Revue d'histoire de l'Amérique française

REVUE D.HISTOIRE DE L'AMÉRIQUE FRANÇAISE

\title{
Économie et société en Nouvelle-France
}

L'historiographie au tournant des années 1960. La réaction à Guy Frégault et à l'école de Montréal — La voie des sociologues

\section{Jean Blain}

Volume 30, numéro 3, décembre 1976

URI : https://id.erudit.org/iderudit/303544ar

DOI : https://doi.org/10.7202/303544ar

Aller au sommaire du numéro

Éditeur(s)

Institut d'histoire de l'Amérique française

ISSN

0035-2357 (imprimé)

1492-1383 (numérique)

Découvrir la revue

\section{Citer cet article}

Blain, J. (1976). Économie et société en Nouvelle-France : l'historiographie au tournant des années 1960. La réaction à Guy Frégault et à l'école de Montréal — La voie des sociologues. Revue d'histoire de l'Amérique française, 30(3),

323-362. https://doi.org/10.7202/303544ar d'utilisation que vous pouvez consulter en ligne. 


\title{
ÉCONOMIE ET SOCIÉTÉ EN \\ NOUVELLE-FRANCE
}

\section{L'HISTORIOGRAPHIE AU TOURNANT DES ANNÉES 1960}

LA RÉACTION À GUY FRÉGAULT ET À L'ÉCOLE DE MONTRÉAL

- LA VOIE DES SOCIOLOGUES -

\author{
JEAN BLAIN \\ Département d'bistoire \\ Université de Montréal
}

Cet article qui fait suite à deux autres ${ }^{1}$ porte sur la façon dont l'image projetée d'un objet historique donné, en l'occurrence la Nouvelle-France, se modifie au cours des années. Les plus récentes études parues en histoire de la Nouvelle-France manifestent l'intention de leurs auteurs de rejoindre les réalités stables, difficiles à cerner, de l'économie et de la société ${ }^{2}$. Dans la perspective de l'historiographie occidentale actuelle, cela n'a rien de très original. Mais, à moins de concevoir l'histoire sous l'angle du progrès continu de la connaissance du passé et en rupture complète avec les idéologies de la société qui cherche à s'interpréter (ce qui nous ramènerait à des temps d'illusions heureusement disparus), il faut convenir que les problématiques et les méthodologies courantes ne peuvent être jaugées que si elles sont insérées dans la trame complexe d'une historiographie donnée qui seule peut en révéler l'à-propos et la valeur³.

1 Jean Blain, «Économie et société en Nouvelle-France. Le cheminement historiographique dans la première moitié du $\mathrm{XX}^{\mathrm{e}}$ siècle", Revue d'histoire de l'Amérique française, 26, no 1 (juin 1972): 3-31 (à l'avenir: Jean Blain, $1^{\mathrm{er}}$ article) et «Économie et société en Nouvelle-France, l'historiographie des années 1950-1960: Guy Frégault et l'école de Montréal", Revue d'histoire de l'Amérique française, 28, no 2 (septembre 1974): 163-186 (à l'avenir: Jean Blain, $2^{\mathrm{e}}$ article).

2 On trouve l'exemple parfait de ces préoccupations dans le livre de Louise Dechêne, Habitants et marchands de Montréal au XVII $\mathrm{e}^{\mathrm{e}}$ siècle (Paris et Montréal, Plon, 1974).

3 On aura compris que dans ces articles, il est surtout question d'une certaine forme d'histoire, disons une macro-histoire, bonne ou mauvaise (l'historiographie se distingue ici de la simple critique des œuvres, une œuvre très déficiente au point de vue de la critique pouvant être très importante au point de vue historiographique.

RHAF, vol. 30, no 3 (décembre 1976) 
En d'autres termes, et pour appliquer ces réflexions à notre sujet d'étude, c'est le point d'arrivée de l'historiographie de la Nouvelle-France, par les problèmes qu'elle a résolus et ceux qu'elle laisse en plan, par les questions nouvelles qu'elle permet d'entrevoir à travers des perspectives idéologiques qui se modifient sans cesse, par les méthodes qu'elle a utilisées, par la quantité et la qualité des sources dont elle s'est servie, bref, c'est cette réalité difficile à analyser qui permet de bien mesurer la pertinence et la portée des œuvres nouvelles qui s'ajoutent, à un moment précis, à la lignée historiographique.

\section{LA NOUVELLE-FRANCE DE GUY FRÉGAULT}

En conclusion d'un article sur le $\mathrm{XVIII}^{\mathrm{e}}$ siècle canadien, Guy Frégault écrivait en 1957:

Il serait inepte de dire - et plus inepte encore de le faire dire à ceux qui ne le disent pas - que le régime français fut l'âge d'or dủ Canada. Le régime français ne fut pas plus l'âge d'or du Canada que l'époque coloniale ne fut l'âge d'or des ÉtatsUnis. Il faut pourtant convenir que, sous le régime français, le Canada connut une évolution normale, tout comme les sociétés contemporaines organisées par la Grande-Bretagne sur le continent américain. En 1760-63, la conquête interrompit le processus de colonisation dans lequel il était engagé. ${ }^{4}$

$\mathrm{Si}$, pour le moment, on laisse de côté l'estocade des premières lignes $^{5}$, on peut convenir que le reste résume fort bien l'aboutisse-

dont l'intérêt vient du fait qu'elle aura pu influencer la vision que les générations ont de leur passé. Ce choix n'est en aucune façon un jugement porté sur des œuvres historiques dont l'objet d'étude est limité et qui peuvent être d'une très grande valeur scientifique.

4 Guy Frégault, "La colonisation du Canada au XVIIIe siècle», Cahiers de l'Académie canadienne-française, 2 - histoire (Montréal, 1957): 79-80. Ce texte est reproduit dans Le XVIII i siècle canadien - Études (Montréal, Éditions HMH, 1968), 385-386.

5 Elle est dirigée contre Fernand Ouellet qui, l'année précédente, avait publié un article intitulé "M. Michel Brunet et le problème de la Conquête», Bulletin des Recherches historiques, 62 (avril-juin 1956): 92-101. Des expressions comme «l'âge d'or du Canada» ou encore «le paradis perdu» appliquées à la NouvelleFrance ont fait fortune, comme stéréotypes commodes chez les adversaires de l'historiographie nationaliste. On les trouve sous la plume non seulement de Fernand Ouellet, mais aussi de Jean-Charles Falardeau, de Guy Rocher, de Fernand Dumont. Si elles correspondent assez bien à l'historiographie groulxiste, elles conviennent mal à l'image que Frégault et l'école de Montréal donnent de la Nouvelle-France. Il s'agit moins pour eux d'un «âge d'or» que de l'embryon d'un «âge d'or» que la Conquête rendra impossible. 
ment de l'itinéraire historiographique de Guy Frégault ${ }^{6}$. "Une évolution normale», affirme-t-il du Canada d'avant la Conquête, qu'estce à dire? L'idée d'évolution démarque assez bien la position des historiens de Montréal au cours de la décennie 1950-1960, de celle de leur maitre, le chanoine Groulx, pour qui l'histoire ressemble davantage à une suite d'instantanés qui fixent pour l'immortalité les faits et les hommes importants, mais surtout les traits caractéristiques du groupe social étudié? de Montréal, c'est la naissance, le développement dans l'existence et le devenir du groupe qui les intéressent.

Cet accent mis sur le dynamisme du groupe social explique la remise à l'honneur, vers 1950, par delà les fresques groulxistes, de l'œuvre d'Émile Salone, déjà vieille d'un demi-siècle, mais qui avait l'avantage de faire de la colonisation le moteur du développement de la société en Nouvelle-France ${ }^{8}$. L'historiographie retournait en arrière pour que l'histoire puisse progresser en se mettant davantage à l'écoute des pulsations économiques et sociales de la réalité coloniale.

Cela dit, le concept d'évolution tel qu'utilisé par Frégault, tout fondamental qu'il est pour l'interprétation globale de l'histoire du Canada que donnera l'école de Montréal, reste un apriorisme qui n'a pas subi l'épreuve de la démonstration historique. Dans des monographies excellentes, Frégault a étudié des pans de la réalité du $\mathrm{XVIII}^{\mathrm{e}}$ siècle canadien. Mais aucun résultat de ces recherches rigoureusement menées n'autorisait à modifier la ligne d'évolution plus diffuse qu'on trouve dans Salone. Quand, dans la brochure de la Société historique du Canada publiée en 1954, Frégault donne de la société canadienne du XVIII ${ }^{\mathrm{e}}$ siècle une image qui n'est plus celle de Salone, une image simplifiée, plus cohérente, plus «logique», une image surtout qui déplace l'accent du paysan au marchand, il succombe à la pression d'un postulat et non à l'évidence de la preuve historique.

\footnotetext{
Voir Jean Blain, $2^{\mathrm{e}}$ article.

Voir Jean Blain, 1er article.

8 En 1976, cette œuvre est toujours considérée comme «l'une des meilleures synthèses de l'histoire socio-économique de la Nouvelle-France». Voir l'introduction par Jean Hamelin de Histoire du Québec (Toulouse, Éditions Privat, 1976). En 1950, Frégault disait à peu près la même chose à ses étudiants. Cet accord à un bon quart de siècle de distance n'implique-t-il pas un certain piétinement de l'historiographie?
} 
D'origine biomorphique, ce postulat assimile la société coloniale à un organisme vivant mis au monde par une métropole (fondations coloniales) et nourri par elle (processus de colonisation) jusqu'à ce qu'il atteigne l'âge adulte (indépendance par consensus ou par révolution) ${ }^{9}$. Ce postulat semble impliquer de plus que les fœtus coloniaux sont anatomiquement et morphologiquement les mêmes dans un cadre chronologique et spatial délimité en gros par l'ère moderne et le monde occidental. D'où la comparaison, toujours a priori, avec la société coloniale américaine; d'où aussi l'idée de «normal» dont l'école de Montréal fait un usage fréquent ${ }^{10}$; société normale, c'està-dire réalité embryonnaire intégrale qui possède dès l'origine toutes les virtualités dont l'actualisation en fera plus tard une société adulte, indépendante, dont le modèle reste les USA d'Amérique; "évolution normale» aussi, pour utiliser les termes de Frégault, c'est-àdire développement de l'organisme sans accident qui en ruine l'intégrité.

On peut dès lors se poser la question suivante: quelle est l'origine de ce postulat? Historiquement, l'embryon colonial comme prototype et comme mesure d'intégrité organique n'a été décrit nulle part, ni en Nouvelle-France, ni dans le monde colonial américain, ni dans le monde colonial espagnol. Il est difficile d'imaginer alors qu'on est parvenu à ce postulat autrement que par référence à une société dite adulte, indépendante de sa métropole, un peu comme on mesure le potentiel d'un enfant aux réalisations d'un homme fait. On perçoit ici, au sein même de la démarche historique, le danger de l'anachronisme et de la logique rétrospective. Car la société dite adulte risque aussi d'imposer comme terme de référence, non seule-

9 Dans les «normes» qu'il utilise pour soń enseignement et qui sont inédites, Maurice Séguin après avoir pris la précaution de dire: «il ne s'agit pas de faire de la société un être réel comme l'individu» et d'affirmer que la vie de société comporte "quelque chose de plus que la vie individuelle» n'en bâtit pas moins tout son raisonnement sur l'analogie entre l'individu et la société. (Voir chapitre premier: «vie et conditions de vie» où les caractéristiques fondamentales de la vie en société sont de pures transpositions de celles de l'individu adulte.) «Vivre pour un individu, écrit Séguin, c'est agir de lui-même, par lui-même, en assimilant les influences, en réagissant à partir de ses propres énergies. La vraie vie individuelle, quoique inséparable du contexte social, est fondamentalement agir propre, autonome, séparation, réaction autour d'un élément personnel qui se réserve.» Plus loin, il ajoute: "Analogiquement, on peut dire à propos des sociétés diverses ce que l'on a affirmé au sujet de l'individu. » (Citations tirées de polycopies avec la permission de l'auteur.)

10 Il serait intéressant de relever la fréquence d'utilisation de cette épithète et de ses équivalents dans les textes de Frégault, Brunet et Séguin. 
ment son caractère d'indépendance politique, mais sa structure de société industrielle, son économie capitaliste moderne, son idéologie nationaliste ${ }^{11}$. À la limite, c'est la société contemporaine avec ses problèmes qui définirait la société coloniale et fournirait toutes les pistes permettant à l'historien d'entreprendre l'étude de celle-ci. Avouons que c'est pousser un peu loin «l'actualité» de l'histoire qui risque alors de verser dans une formidable tautologie. Remarquons aussi que c'est tomber dans un travers semblable à celui que Frégault et les siens reprochaient à leurs devanciers, c'est-à-dire de concevoir avec nostalgie la Nouvelle-France comme la réalisation à une époque lointaine de l'idéologie sociale - agriculturiste, etc. - qu'ils poursuivaient à la fin du XIX ${ }^{\mathrm{e}}$ et au début du XX $X^{\mathrm{e}}$ siècle. Entre l'école de Montréal et les historiens qui ont précédé, il y a un rajustement d'idéologies qu'expliquent la prise de conscience et l'acceptation de vivre dans un monde urbanisé et industriel. Entre la Nouvelle-France de Groulx et celle de Frégault, il y a le demisiècle qui sépare la vision du monde de 1910 de celle de 1960. Mais le processus de l'historien qui consiste à projeter - peut-être un peu trop lourdement - son présent dans le passé reste le même.

Dernier trait de cette Nouvelle-France, enfant de métropole, organiquement bien conformé, promis dès le berceau à l'indépendance de l'âge adulte où sa bourgeoisie capitaliste perçue de l'observatoire du $\mathrm{XX}^{\mathrm{e}}$ siècle pourra alors donner toute sa mesure, elle développe dès le début ses innéités de société nationale distinguant petit à petit les siens des «autres» : forains, métropolitains, anglais...

À vrai dire, cette caractéristique n'est pas nouvelle. Elle marque l'historiographie canadienne depuis déjà un bon siècle. Frégault rejoint Garneau en donnant la main au chanoine Groulx. Par delà les divergences nées de l'accent qu'on a pu mettre sur les conditions d'existence du groupe social, alors qu'on les mettait sur ses qualités d'être; par delà l'inversion qui fait de la Nouvelle-France une société mercantile à base paysanne, alors qu'on en faisait une société paysanne avec des appoints mercantiles, tous ces historiens s'accordent pour laisser entendre que cette société à l'époque de la Nouvelle-France a tendance à se percevoir globalement par opposi-

11 Ce procédé est particulièrement visible chez Michel Brunet, dans «La Conquête anglaise et la déchéance de la bourgeoisie canadienne (1760-1793)", article publié dans Amérique française en juin 1955 et reproduit dans La présence anglaise et les Canadiens, études sur l'histoire et la pensée des deux Canadas (Montréal, Beauchemin, 1958), 49-112. 
tion à ce qui n'est pas elle culturellement. Constatons simplement ici qu'un tel procédé insère dans l'embryon du XVII ${ }^{\mathrm{e}}$ siècle le germe vivant du nationalisme qui, idéologiquement, fleurit au XIX ${ }^{\mathrm{e}}$ siècle. Constatons aussi que cette enveloppe nationale tient lieu d'enveloppe sociale, de telle sorte que le traitant ou le paysan de Nouvelle-France ne sont jamais conçus d'abord comme traitant ou paysan, mais comme «canadien» s'occupant de commerce ou d'agriculture. Constatons enfin que cette conception nationaliste de l'histoire présuppose déjà le désastre de la conquête dans la mesure où celle-ci signifie la domination et la présence continue de l' "autre»: désastre d'ordre culturel selon la vieille historiographie, désastre d'ordre existentiel et organique selon Frégault et les historiens de l'école de Montréal.

En 1763, la Grande Bretagne prit le Canada. Elle le prit pour en tirer parti et non pour servir la population autochtone. Dans sa nouvelle colonie, il était naturel qu'elle fit une politique britannique, qu'elle instaurât une économie britannique et qu'elle favorisât i'épanouissement d'une coilectivité britannique. Les «anciens sujets» qui vinrent s'établir dans la province de Québec (nom officiel du Canada vaincu) furent en mesure de dominer la société défaite des Canadiens parce qu'ils purent seuls profiter de la substitution de colonisation provoquée par la conquête. ${ }^{12}$

Au moment où Frégault rédige ces lignes, ce que l'on a nommé la «révolution tranquille» ourdit ses complots un peu partout contre le duplessisme vieillissant; la première ligne d'attaque qui claironne son antinationalisme sort des officines de Cité Libre et du giron du père Lévesque. Elle se bat au nom d'une vision nouvelle de la société qui implique la remise en question - à partir d'une sociologie devenue rigoureuse - de l'explication des historiens nationalistes de tout acabit. Par ailleurs, au même moment, au plan du métier d'historien, des problématiques empruntées des historiographies de pointe, impliquant une vision infra-nationale des réalités sociales et proposant l'utilisation de méthodes et de sources nouvelles, commencent à percer au Québec.

Quel sera le sort de cette Nouvelle-France redevenue objet de colonisation comme au temps de Salone et dont Frégault a contribué à modifier l'image, une Nouvelle-France désormais dépeuplée 386.

12 Guy Frégault, "La colonisation du Canada au XVIII ${ }^{\mathrm{e}}$ siècle», op. cit.: 
de ses héros, délestée de son mysticisme et moins marquée par la tranquille possession de ses valeurs culturelles; une Nouvelle-France engagée très tôt dans le développement «normal» de sa société, animée par les objectifs de gain mercantile plutôt que par ceux de l'établissement paysan, dominée moins par son clergé et ses seigneurs que par sa classe d'entrepreneurs qui préfigurent déjà ce qu'ils seraient devenus à l'ère du chemin de fer et de l'industrialisation n'eussent été la Conquête et l'occupation permanente des Britanniques; une Nouvelle-France surtout qui, à travers tous ces changements historiographiques, reste prisonnière de l'identité nationale qu'ont tissée autour d'elle tous les historiens depuis Garneau?

\section{LES SOCIOLOGUES ET L'HISTOIRE NATIONALISTE}

Racontant avec un brin de complaisance les «antécédents, débuts et croissance de la sociologie au Québec», Jean-Charles Falardeau écrit:

La nature des enseignements et l'orientation des recherches des sociologues laissent suffisamment entendre ce qu'ont été les formes de leurs interrogations et de leurs analyses de la société québécoise. Elles signifient aussi qu'ils ont très tôt et intimement participé à la vie de cette société, dont, à leur gré, les perceptions qu'elle avait d'elle-même et les idéologies correspondaient de moins en moins à la réalité. ${ }^{13}$

Cette participation qui prend la forme d'une contestation et «qui a plus que préparé, façonné la 'révolution tranquille' de 1960 » se situe entre les années 1952 et 1962 . Bien entendu, les sociologues ne sont pas seuls dans la mêlée. Avec eux, on trouve d'autres universitaires, des représentants des mouvements syndicaux, et «une jeune intelligentsia d'animateurs de discussions et de commentateurs à la chaîne française de Radio-Canada - dont plusieurs étaient d'anciens étudiants en sciences sociales de Laval». Bref, des sociologues et beaucoup de gens frottés de sociologie. Ce qu'ils contestent? Avant tout, «l'immobilisme idéologique» qui "n'était que trop satisfait de se perpétuer et de se justifier en s'alimentant aux sources du corporatisme et du slogan toujours populaire pro-

13 Jean-Charles Falardeau, «Antécédents, débuts et croissance de la sociologie au Québec», Recherches sociographiques, XV, nos 2-3 (mai-août 1974): 156. Ce numéro fut réimprimé sous le titre, La Sociologie au Québec (Québec, Les presses de l'Université Laval, 1975). 
clamant la primauté de la vie agricole», et "la rhétorique autonomiste» qui "flattait en le soporifiant le sentiment aussi populaire d'un nationalisme qui s'opposait pour des raisons plus ou moins ambiguës au gouvernement fédéral ${ }^{14}$.

Il va de soi que cette "rhétorique autonomiste» et soporifique englobe le discours historique tel que le perçoivent les sociologues des années 1960. Du reste, citant «certains des textes sociologiques qui ont le plus contribué à opérer le décrochage avec les idéologies dominantes », Falardeau mentionne trois «articles percutants» parus dans Cité Libre et qui tous trois touchent, d'une façon plus ou moins directe, à l'historiographie. Ce sont: de Marcel Rioux, «Idéologie et crise de conscience du Canada français " ${ }^{15}$, de Léon Dion, "Le nationalisme pessimiste: sa source, sa signification, sa validité ${ }^{16}$, de Fernand Dumont, «De quelques obstacles à la prise de conscience chez les Canadiens français ${ }^{17}$.

Il faut convenir que ces textes, à l'exception de celui de Léon Dion, ne concernent notre propos que par le biais, ce quii n’implique pas pour autant que leur influence ait été négligeable sur l'historiographie du Canada et de la Nouvelle-France. Implicitement ou explicitement, ils réclament - et en ce sens ils font chorus avec beaucoup d'autres textes de l'époque - la venue d'une nouvelle histoire qui ne soit plus «systématisée» mais polyvalente, qui ne soit plus l'expression d'un nationalisme conçu par «la bourgeoisie des professions libérales », bref, continue Dumont:

Il faut qu'on nous donne une autre histoire qui ne nous apprenne pas seulement que nos Pères ont été vaincus en 1760 et n'ont plus fait ensuite que défendre leur langue... une histoire qui ne masque plus la naissance de notre prolétariat par un chapitre sur les écoles séparées. ${ }^{18}$

Deux mois auparavant, Dion terminait son article en disant:

Il est temps qu'à côté de l'histoire nationale s'élabore une histoire sociale qui reprenne sous de nouveaux angles, l'expérience politique, économique et culturelle des Canadiens français en tant qu'ils furent des ouvriers, des paysans et des bourgeois, c'est-à-dire des hommes qui ont travaillé et cherché à se définir par rapport aux structures concrètes à l'intérieur desquelles

14 Ibid. : 156-157.

15 Cité Libre, 14 (décembre, 1955): 1-29.

16 Cité Libre, 18 (novembre, 1957): 3-18.

17 Cité Libre, 19 (janvier 1958): 22-28.

18 Ibid. : 28. 
leur existence prenait virtuellement son sens et qui, sans doute, ont aspiré vers certaines formes de libération et de transcendance. ${ }^{19}$

Après plus d'un siècle d'histoire fabriquée selon la ligne du développement national, l'appel des sociologues arrivait sans doute à son heure et bien qu'il soit difficile de le démontrer, il est plus que probable qu'il ait contribué au changement d'orientation historiographique qu'on constate après 1960 .

Cela dit, on ne peut s'empêcher de remarquer qu'il est lancé dans un climat de confusion, d'illogisme, voire d'ignorance, et que, dès lors, il prend volontiers la forme de la systématisation qu'il reproche justement à ce qu'il combat. Signalons que ces textes, qui ont une incontestable valeur existentielle, (pour utiliser une expression chère aux sociologues) font partie de l'arsenal utilisé entre 1950 et 1960 (et dont les pièces d'artillerie sont Cité Libre ${ }^{20}$, La Grève de l'Amiante ${ }^{21}$, Essais sur le Québec contemporain ${ }^{22}$, La dualité canadienne ${ }^{23}$, l'Institut canadien des affaires publiques ${ }^{24}$ ) pour com-

19 Léon Dion, «Le nationalisme pessimiste, sa source, sa signification, sa validité », Cité Libre, 18 (novembre 1957): 18.

20 «La revue Cité Libre avait été fondée en 1950 par Gérard Pelletier, PierreElliott Trudeau et Jacques Hébert, sous l'inspiration de la philosophie personnaliste de Mounier et de la revue Esprit. Elle se préoccupe d'actualiser dans notre société redevenue de type mérovingien les préoccupations démocratiques et civiques, le sens du temps présent. » Jean-Charles Falardeau, «Antécédents, débuts et croissance de la sociologie au Québec», Recherches sociographiques, op. cit. : 157.

21 En collaboration et sous la direction de Pierre-Elliott Trudeau, La Grève de l'amiante (Montréal, les éditions Cité Libre, 1956). L'ouvrage est resté célèbre à cause de son premier chapitre: "La province de Québec au moment de la grève», rédigé par Trudeau lui-même. Bel exemple de simplification hâtive pour donner du poids à ce que l'on veut prouver.

22 Édités par Jean-Charles Falardeau. Essais sur le Québec contemporain Essays on Contemporary Quebec (Québec, Canada, Les presses universitaires Laval, 1953). Le texte le plus percutant est celui de Maurice Tremblay: «Orientations de la pensée sociale», 193-208.

23 Canadian Dualism, Studies of French-English Relations. La dualité canadienne, essais sur les relations entre Canadiens français et Canadiens anglais, edited by Mason Wade (Toronto, Québec, University of Toronto Press, Presses universitaires Laval, 1960). Rappelant le discours présidentiel de Gustave Lanctôt à la Société royale du Canada en 1949, Jean-Charles Falardeau, en avant-propos, déclare: «Il souhaitait que le Canada cessât d'être le pays de «deux solitudes» pour devenir, en exemple au monde, le pays de «deux fortitudes». Peut-être sommes-nous déjà engagés dans cette nouvelle étape. Est-il téméraire d'espérer que ce livre, à sa façon, éclairera la route dans cette direction?».

24 Fondé en 1954, «ses congrès annuels d'octobre, à Sainte-Adèle, devinrent vite la contrepartie laïque et chirurgicale des somnolentes Semaines sociales». dit 
battre le nationalisme canadien-français sous toutes ses formes, nationalisme de faits, de comportements, d'idéologies. Compte tenu de l'objectif premier à atteindre, le changement de régime politique au Québec, on ne s'étonne guère que ce blitz, même sous des dehors savants et dans un langage parfois technique, n'ait pas fait de la sérénité sa première vertu, ni qu'il ne se soit laissé empêtrer dans les nuances.

\section{Idéologie monocorde et historiographie déracinée}

Aussi, tire-t-on à boulets rouges sur «l'idéologie canadiennefrançaise» sans le moindrement mettre en doute le caractère d'unicité qu'on lui prête. Marcel Rioux écrit:

Au Canada français, il y a toujours eu une idéologie. Depuis 1760 , elle n'a guère varié. On peut soutenir qu'elle s'est figée après la Conquête et que, tout au long de notre histoire, elle n'a guère varié que dans sa formulation. L'idéologie canadiennefrançaise s'est toujours appuyée sur trois caractères de la culture canadienne-française: minoritaire, catholique et française. C'est à partir de ces caractères, envisagés d'abord dans le concret, mais avec les années de plus en plus schématiquement que l'idéologie a formé sa doctrine nationale et qu'elle en est arrivée à contrôler la pensée et la plupart des institutions éducationnelles et intellectuelles du Québec. ${ }^{25}$

Notons un procédé semblable chez Dumont ${ }^{26}$ avec cette différence que l'idéologie nationaliste, tout aussi monolithique, remonterait à la crise de conscience de la bourgeoisie de 1840. Quelques années plus tard, dans un texte remarquable intitulé: «L'étude systématique de la société globale canadienne-française», Dumont luimême avouera qu' "on n'a pas considéré suffisamment les idéologies comme des phénomènes spécifiques; le plus souvent, poursuit-il, la définition explicite ou implicite des auteurs s'élargit à toute la culture canadienne-française ou encore désigne un ensemble d'attitudes confuses». Et il conclut: «les études de nos idéologies devraient donc réduire beaucoup plus strictement leur objet ${ }^{27}$.

encore Falardeau. "Antécédents débuts et croissance de la sociologie au Québec", Recherches sociographiques, op. cit.: 158.

${ }^{25}$ Marcel Rioux, «Idéologie et crise de conscience du Canada français», Cité libre, 14 (déc. 1955): 9-10.

26 Fernand Dumont, «De quelques obstacles à la prise de conscience chez les Canadiens français », Cité Libre, 17 (janv. 1958): 27.

27 Fernand Dumont, "L'étude systématique de la société globale canadiennefrançaise», Situation de la recherche sur le Canada français (Québec, les Presses de l'Université Laval, 1962): 289-290. 
C'était, en 1962, à une époque où la sérénité revenant peu à peu aux esprits, on commençait à introduire des nuances là où on n'en faisait guère encore quelques années auparavant. Dans ce même texte, Dumont écrivait, à propos de l'historiographie:

Une autre coupure structurelle doit être signalée: celle que présente un groupe d'historiens de l'Institut d'histoire de l'Université de Montréal. Ils sont nationalistes, mais avec des colorations importantes qui les distinguent de leurs prédécesseurs - du chanoine Groulx, en particulier. Pour eux, la Nouvelle-France avait donné lieu à une structure sociale complète dont le dynamisme était représenté par une bourgeoisie locale importante. La Conquête est venue bouleverser ces assises de notre nationalité. ${ }^{28}$

Mais avant 1960, c'est assez curieusement en jaugeant la teneur en «pessimisme» et en «optimisme» que les sociologues distinguaient - ou ne distinguaient pas - les courants historiographiques nationalistes. Dans son «Idéologie et crise de conscience du Canada français», Marcel Rioux note bien que "pour certains historiens, ce sont les centralisateurs canadiens qui ont ourdi un noir complot afin d'assimiler la nationalité canadienne-française», alors que «d'autres, plus perspicaces et plus érudits, remontent à la défaite de 1760 et y voient la cause de tout notre mal». Mais, négligeant les implications pourtant énormes de ce qu'il venait d'affirmer, il conclut aussitôt: «les uns comme les autres sont d'un pessimisme noir et s'accordent à penser que, cette fois-ci, nous surmonterons bien difficilement les obstacles ${ }^{29}$.

Trois ans plus tard, en 1958, dans un article intitulé «Les Canadiens français et leur idéologie», Jean-Charles Falardeau apporte son propre témoignage pour renforcer la démonstration: "l'idéologie nationaliste», dit-il, inspire les «travaux de la jeune école d'historiens montréalais dont le pessimisme et le défaitisme font parầtre, à distance, l'œuvre du chanoine Groulx comme une symphonie de sérénité ${ }^{30}$. C'était quelque temps après la parution dans Cité Libre du texte de Léon Dion déjà cité: "Le nationalisme pessimiste. Sa source, sa signification, sa validité».

$28 \quad$ Ibid.: 284.

29 Marcel Rioux, «Idéologie et crise de conscience du Canada français», Cité libre, 14 (déc. 1955): 2.

30 Jean-Charles Falardeau, «Les Canadiens français et leur idéologie», La dualité canadienne (1960): 34 . 
Certes, il paraît bien étrange aujourd'hui qu'on ait pu identifier un courant historiographique en se préoccupant beaucoup plus de l'impression qui se dégageait des conclusions des ouvrages que de la problématique que ceux-ci mettaient en œuvre. Mais le procédé s'explique si on considère la dialectique de base des sociologues et autres contestataires de la décennie 1950-1960. Il s'agissait d'abord, comme on l'a vu, de faire état d'une idéologie systématisée, unique, sclérosée, idéologie qu'on qualifie de clérico-nationaliste. Par la suite, on affirmait que l'historiographie constituait l'un des principaux véhicules de cette idéologie. Le formel de la preuve - et ce n'était pas toujours commode - consistait à montrer alors l'écart (le caractère non-existentiel) qui existait entre l'idéologie et les diverses couches de la société en pointant du doigt l'irréalisme, non pas du discours historique lui-même (ce que les sociologues auraient eu de la difficulté à faire), mais l'irréalisme des appels qu'il contenait implicitement ou explicitement, appels à des actions concrètes, à des comportements, à des attitudes d'esprit. Voilà ce qui soustend, par exemple, le fameux texte de P.-E. Trudeau dans la Grève de l'Amiante. Rioux pour sa part fait un usage copieux de ce procédé. «Il ne semble pas, dit-il, que le Québécois souffre indûment d'infériorité ni qu'il soit exagérément nationaliste... L'étude objective de la société québécoise porte à croire que la majorité des individus loin d'être dans un état de prostration, sont dynamiques, confiants et peu embarrassés de leurs voisins... Sur le plan technique et économique, le Québécois, malgré de forts handicaps, créés en partie par l'idéologie, est aussi avancé que plusieurs de ses voisins des autres provinces; libéré de la camisole de force, de «notre doctrine nationale», le Québécois peut prendre sa place avantageusement parmi les autres citoyens du monde ${ }^{31}$.»

Plus subtil, Dumont tout en s'en prenant lui aussi à «la coque mythique standardisée» de l'idéologie nationaliste qui ne permet pas «une diversification des fidélités au passé »et colle mal à la réalité, flaire pourtant le piège qu'il y a à la rejeter en bloc et à tenter «de passer directement à l'humain, sans médiation par la culture ${ }^{32}$.

Léon Dion reprend à son tour le même procédé, la prudence en moins. Mais une difficulté surgit: comment démontrer le caractère

\footnotetext{
31 Marcel Rioux, "Idéologie et crise de conscience du Canada français», Cité libre, 14 (déc. 1955): 21-22.

32 Fernand Dumont, "De quelques obstacles à la prise de conscience du Canada français, Cité libre, 19 (janv. 1958): 25.
} 
non existentiel des positions de Frégault et de Brunet? On ne peut certes plus parler ici des rêves chimériques de retour à la terre, de corporatisme, de ligues d' "achat chez nous». On ne peut parler de crainte maladive de l'État, ni de soumission aveugle à la doctrine sociale de l'Église. Qu'à cela ne tienne, on prouvera l'irréalisme de l'école néo-nationaliste par son "pessimisme», étant sous-entendu que les peuples n'ont jamais l'instinct suicidaire. De là, la fortune du vocable que les sociologues se sont transmis l'un à l'autre ${ }^{33}$ et qui permettait de distinguer deux courants historiographiques et de les condamner rapidement ensuite sous prétexte que l'un et l'autre n'avaient aucune résonance dans la réalité sociologique. Parlant de l'école de Montréal, Dion écrit:

On voit très clairement dans la divergence entre l'état de pessimisme chronique de l'historiographie néo-nationaliste et la façon plutôt pragmatique dont les groupes sociaux, notamment la bourgeoisie, à partir de l'expérience de leur situation se conçoivent et perçoivent leur avenir que l'historiographie actuelle, dans sa double modalité séculière et nationaliste, s'élabore tout à fait en dehors de la structure sociale à partir de laquelle les groupes sociaux canadiens-français se définissent concrètement. ${ }^{34}$

Diagnostic intempestif et malencontreux, s'il en fut, ainsi que le prouve l'histoire du Québec depuis la «révolution tranquille», avec la montée des mouvements indépendantistes et la trouée qu'ils ont pratiquée au cœur même des vieilles institutions fédéralisantes québécoises, politiques et sociales. Il n'est pas étonnant qu'on ait, depuis ce temps, entendu d'aussi nombreuses palinodies chez ceuxlà mêmes - sociologues ou autres définisseurs de situation - qui

33 On décide alors que le vocable caractérisait tout particulièrement l'école de Montréal. Selon Dion, c'est Albert Faucher qui aurait eu la paternité de cette trouvaille à l'occasion d'un cours professé à l'Université de Toronto en 1957, sous les auspices des "Grey Lectures». Voir Léon Dion, "Le nationalisme pessimiste, sa source, sa signification, sa validité», Cité libre,: 3. "L'accusation de pessimisme est tout à fait ridicule. Dit-on d'un médecin que son diagnostic est optimiste ou pessimiste? La question est de savoir s'il a raison ou s'il se trompe», Michel Brunet, «La science historique au Canada français: son évolution et ses développements actuels ", Québec-Canada anglais, deux itinéraires un affrontement (Montréal, les éditions HMH, 1968), 40. D'ailleurs, Michel Brunet se donnait lui-même comme la preuve vivante, bien portante, de la fausseté de l'expression.

34 Léon Dion, "Le nationalisme pessimiste. Sa source, sa signification, sa validité », Cité libre, 18 (nov. 1957): 14. Il continue: "Une historiographie qui ne parvient pas à établir un lien positif, réel ou actuel entre un peuple et son passé, doit être abandonnée ou ignorée parce qu'elle n'a pas de sens légitime.» 
avant 1960 menaient le combat antinationaliste ${ }^{35}$. «Science des phénomènes sociaux, la sociologie est aussi conscience de la société ", déclare Falardeau ${ }^{36}$. Il faut bien admettre aujourd'hui que dans les années 1950-1960, cette conscience y voyait confusément, prise qu'elle était d'attaques virulentes d'antiduplessisme aigu.

\section{Vision anachronique et solutions artificielles}

À la décharge de Léon Dion, convenons qu'il n'avait pas encore en mains, en 1957, toutes les pièces du dossier de l'historiographie néo-nationaliste, et que celles qu'il possédait n'étaient peutêtre pas de nature à l'écarter du cul-de-sac du "pessimisme» outrancier. Par ailleurs, il fut bien l'un des premiers à saisir le caractère radical du nationalisme de l'école de Montréal à travers une démonstration historique qui, il faut l'avouer, laissait à l'époque grandement à désirer, ne comportant en fait que les extrapolations de Frégault à partir d'ouvrages sur la Nouvelle-France et les études de Brunet qui s'attachaient moins au développement de la société canadienne après la Conquête qu'à l' «histoire de la pensée des deux Canadas ${ }^{37}$.

Léon Dion part d'un critère fort discutable (en tout cas partiel) de distinction entre Groulx et les historiens de l'école de Montréal.

Il est impossible de comprendre les néo-nationalistes si l'on ne voit pas d'abord en eux des historiens «laïcs»; de même on ne peut comprendre Groulx si on ne reconnaît pas, derrière l'écrivain, le « clerc». ${ }^{38}$

C'était donner de l'œuvre de Groulx et de l'abondante historiographie groulxiste une image déformée où les motifs $\mathrm{d}$ ' "optimisme" paraissaient étroitement liés au «rôle éminent tenu par l'Église» dans l'histoire nationale et à l'assurance de sa pérennité, et de son indéfectibilité. Ce n'est là, chez Groulx, qu'un aspect d'une vision

35 On se reportera aux itinéraires intellectuelles et politiques de Rioux, Dumont et Dion.

36 Jean-Charles Falardeau, «Antécédents, débuts et croissance de la sociologie au Québec», Recherches sociographiques, XV, no 2-3 (mai-août 1974): 135.

37 Jean Blain, "Maurice Séguin ou la rationalisation de l'histoire nationale», préface de Maurice Séguin, La «nation canadienne» et l'agriculture (1760-1850). Essai d'histoire économique (Trois-Rivières, Le Boréal Express Ltée, 1970), 35.

38 Léon Dion, «Le nationalisme pessimiste. Sa source, sa signification, sa validité», Cité libre, 18 (nov. 1957): 5. 
qui englobe toute la réalité de l' "être» national: sa foi, mais aussi sa langue, son droit, sa culture, ses traditions, son histoire et enfin toutes les institutions sur lesquelles ceux-ci trouvent appui pour se maintenir ${ }^{39}$. En revanche, Dion a bien raison d'affirmer que «dans la perspective séculière [laïque] du national, il n'y a qu'un rêve qui puisse contenir tout entier les aspirations de la nation: le rêve de l'État». Mais alors pourquoi, pour un observateur de 1957, ce rêve paraît-il à ce point irréalisable et pourquoi, selon lui, confine-t-il au «pessimisme» le plus noir? Dion se laisse prendre par l'accent indu que les néo-nationalistes, à l'origine, ont placé eux-mêmes sur la bourgeoisie et l'entrepreneurship en tentant de démontrer leur thèse, et qui a été, par la suite, fortement atténué du moins chez Séguin ${ }^{40}$. Par ailleurs, Dion ne paraît connaître de nationalismes triomphants que ceux du XIX ${ }^{\mathrm{e}}$ siècle qui s'appuyaient sur des bourgeoisies libérales. Il a beau jeu alors d'écrire:

...la perspective séculière du néo-nationalisme ne peut aboutir qu'au pessimisme et au défaitisme, tant, en tout cas, que l'historiographie s'élaborera sur les indices incertains et insatisfaisants dont on dispose actuellement sur le caractère véritable, l'orientation et les aspirations effectives de la bourgeoisie capitaliste canadienne-française. ${ }^{41}$

Plus attentif au mouvement général de décolonisation qui faisait rage pourtant au moment même où il écrivait, Dion y aurait peutêtre trouvé la relation qu'il cherchait entre le rêve néo-nationaliste et la réalité sociologique québécoise. Et l'argument du "pessimisme» y aurait par contre perdu à ses yeux beaucoup de sa valeur démonstrative.

Il faudra attendre l'année 1962 (après la chute de Duplessis) pour que les sociologues, par la voix de Fernand Dumont, commencent à percevoir le caractère «existentiel» des positions de l'école de Montréal.

39 Jean Blain, 1er article.

40 Pour Séguin, l'état national est le garant d'une société qui peut être aussi bien socialiste que capitaliste. L'accent sur la bourgeoisie et l'entrepreneurship qu'on trouve surtout dans les premiers ouvrages de l'école de Montréal, vient d'abord de l'explication historique qu'on a tenté de donner de la société du $\mathrm{XIX}^{\mathrm{e}}$ et du début du XX $\mathrm{X}^{\mathrm{e}}$ siècle. Il vient aussi de l'impossibilité pour un Québécois avant 1960 - et cela est visible aussi chez les sociologues contestataires - de concevoir une société nord-américaine qui ne serait pas capitaliste.

41 Léon Dion, «Le nationalisme pessimiste. Sa source, sa signification, sa validité », Cité libre, 18 (nov. 1957): 13. 
Cette école historique a apporté beaucoup à la définition de notre société. Alors qu'elle semble nous emprisonner tragiquement dans un passé assez lointain, les questions qu'elle pose sourdent de nos problèmes les plus actuels, malgré les apparences, c'est encore le présent (et il en est toujours fatalement ainsi pour l'historien) qui est au départ de l'investigation. En effet, c'est une constatation des faillites des idéologies traditionnelles, de leur peu de réalisme devant nos problèmes d'aujourd'hui qui différencie, en définitive, les travaux de M. Groulx et ceux de l'école dont nous parlons. On retrouve ainsi un accord profond avec d'autres courants de pensée non nationalistes: la dénonciation des mythes et du patriotisme sentimental n'est pas moins vive chez les uns que chez les autres. Tout en étant, sous bien des aspects, les héritiers de la problématique traditionnelle de notre historiographie, ces jeunes historiens, témoignent à leur manière, de sa faillite. Enfin, il serait tout à fait ridicule de nier la très grande importance de la Conquête dans l'édification de notre société : en ce sens, pour reprendre une expression familière aux sociologues, on peut dire que cette école a construit un modèle partiel parfaitement valable de notre société globale. ${ }^{42}$

Modèle partiel bien sûr, d'ailleurs beaucoup trop simple pour ne pas être partiel, mais qui avait quand même l'avantage de la cohérence et de la logique interne. On ne pouvait en dire autant des conclusions auxquelles avait abouti le raisonnement des sociologues qui s'en prenaient aux idéologies et à l'historiographie nationalistes. Ils ne paraissaient avoir d'autres choix que la naïveté ou l'illogisme. Dans le premier cas, ils courcircuitaient sans ambage le milieu social pour rejoindre l'humain. Ainsi Marcel Rioux qui réclame une gauche canadienne-française, «parce que la gauche, écrit-il, comme le dit Malraux, se bat toujours pour des principes universels valables pour tous les hommes parce qu'elle envisage d'abord l'homme, l'être humain avant le Turc ou le Bulgare ${ }^{43}$. Dans le second cas, après avoir combattu l'idéologie nationaliste, globalement montré son étroitesse, son manque de concordance avec la réalité sociale, ils en arrivaient à demander qu'on troque un nationalisme pour un autre, sans trop comprendre que, par là même, ils se réinséraient dans le courant le plus fort, le plus triomphant de l'idéologie qu'ils dénonçaient: «La définition que le Canada donne maintenant de lui-même, écrit Falardeau, proclame ce jumelage [sic] de deux grands groupes do-

42 Fernand Dumont, "L'étude systématique de la société globale canadiennefrançaise", Situation de la recherche sur le Canada français, op. cit.: 284.

43 Marcel Rioux, «Idéologie et crise de conscience du Canada français», Cité libre, 14 (déc. 1955): 29. 
minants. Pour que cet équilibre se stabilise, il requiert la présence franche de chacun des deux protagonistes qui ont maintenant prouvé qu'ils «se sont reconnus, qu'ils s'acceptent et qu'ils s'entr'aident l'un l'autre ${ }^{44}$. On croirait entendre sir Thomas Chapais. Dion, pour sa part, y va de la formule interrogative: «L'échec des néo-nationalistes s'expliquerait-il par le fait que ceux-ci se sont trompés sur la réalité de l'objet national canadien-français, cet objet devant se définir aujourd'hui par référence non pas au Canada français mais au Canada tout entier?» Heureusement, l'hypothèse, encore qu'elle fût séduisante, ne lui paraissait pas tout à fait fondée. Restait alors l'autre possibilité, celle de l'accession à une culture fondée sur l'internationalisation: "On voit s'accréditer cette opinion que l'ancien idéal d'une possession exclusive, pour chaque peuple, des outils de la culture (en particulier les institutions économiques et politiques) doit être délaissé; au contraire, on voit bien qu'il faut parfois les distribuer en propriété conjointe afin que chaque peuple, étant plus riche par la mise en commun des ressources et l'internationalisation de certaines fonctions politiques, puisse en faire bénéficier plus largement la culture propre qu'a édifiée pour lui l'histoire ${ }^{45}$.»

Solutions artificielles, irréalistes; l'une, objecte déjà Dumont en 1958, fabriquera des «hommes de nulle part». L'autre nous gratifiera «d'un mythe supplémentaire», appelé «la nation canadienne ${ }^{46}$.

Dans cette aventure exaltante qui mena au déblocage des années 1960 , l'historiographie d'alors, prise à parti directement ou indirectement, s'en sortait finalement à peu près indemne surtout à cause de la faiblesse, du manque de nuances et de l'incohérence de la démarche intellectuelle de ses contradicteurs. Elle sera bien davantage affectée par le climat de conflit où la neutralité et les distinguos devenant impossibles, il lui faudra se résigner à être confondue dans une opposition rétrograde à abattre. C'est en ce sens que les sociologues et en général les hommes de gauche de cette époque avec leur appel (qu'on serait tenté de dire plus instinctif que raisonné) à l'histoire sociale, ont pu préparer le terrain à un changement des problématiques historiques.

\footnotetext{
44 Jean-Charles Falardeau «Les Canadiens français et leur idéologie», $\mathrm{La}$ dualité canadienne, op. cit.: 38 .

45 Léon Dion, «Le nationalisme pessimiste, sa source, sa signification», Cité libre, 18 (nov. 1957): 16 et 17.

46 Fernand Dumont, "De quelques obstacles à la prise de conscience chez les Canadiens français », Cité libre, 19 (janv. 1958) : 24.
} 


\section{LES SOCIOLOGUES ET LA NOUVELLE-FRANCE}

Entre-temps et quoi qu'ils en aient, les sociologues sont bien obligés de se contenter des visions du passé que leur ont léguées les historiens. Et il est étonnant de constater jusqu'à quel point ces visions pourtant décriées ont pu marquer non seulement leurs œuvres, mais certaines orientations de la sociologie québécoise durant la décennie 1950-1960. Ce n'est pas le lieu ici d'étudier dans toute son ampleur le problème de filiation - consciente ou inconsciente - entre l'appréhension historique des réalités sociales souvent empruntée à une historiographie vieillotte et le travail formel du sociologue ou d'une façon plus générale du spécialiste des sciences sociales. Nous nous contenterons, pour rester dans le cadre de notre sujet, de voir de quelle image globale de la Nouvelle-France, parfois présupposée, parfois partiellement décrite, ceux-ci se sont servis pour étayer leurs enquêtes ou leurs analyses ${ }^{47}$.

\section{Léon Gérin: une certaine vision historique ${ }^{48}$}

Tous les sociologues s'entendent pour reconnaître le rôle pionnier joué par Léon Gérin dans l'application, à un champ québécois, des méthodes de la "science sociale ${ }^{49}$. Jean-Charles Falardeau a raconté avec détails et admiration l'itinéraire intellectuel de Gérin ${ }^{50}$. C'est à lui que nous empruntons les quelques données qui suivent. Parti à Paris à l'automne de 1885 pour y étudier la botanique et la biologie, Gérin se retrouve à l'École de la science sociale que dirigent deux disciples de Le Play: Henri de Tourville et Edmond Demolins. Il en revient au printemps de 1886 avec un bagage de connaissances nouvelles et beaucoup d'enthousiasme.

47 Il est entendu que les propos qui suivent ne constituent en aucune façon une critique des démarches sociologiques proprement dites. Ils ne concernent que les appuis historiques grâce auxquels les sociologues ont l'habitude d'en présenter les résultats dans un certain devenir.

48 Jean Blain, $1^{\mathrm{er}}$ article, note 55.

49 Fernand Dumont, "L'étude systématique de la société globale canadiennefrançaise», Situation de la recherche sur le Canada français, op. cit: 285 . - JeanCharles Falardeau, "Antécédents, débuts et croissance de la sociologie au Québec», Recherches sociographiques, XV, nos 2-3 (mai-août 1974): 138. - Hervé Carrier, Le sociologue canadien Léon Gérin, 1863-195I (Montréal, Éditions Bellarmin, 1960), 9-10.

so Jean-Charles Falardeau, "Présentation de Léon Gérin et de son œuvre», dans Léon Gérin et l'habitant de Saint-Justin (Montréal, les Presses de l'Université de Montréal, 1968), 11-48. Ces textes de J.-C. Falardeau, d'abord parus dans Recherches sociographiques (vol. 1, 2 et 4), datent de 1960-1963. 
Dès son retour, Gérin se met à l'œuvre. Déjà en 1886 il est à Saint-Justin où il commence une enquête, selon la méthode de l'École de la science sociale, qui aboutit à sa célèbre monographie de «l'habitant de St-Justin» livrée, dans sa version finale, en 1898, dans les Mémoires de la Société royale du Canada ${ }^{51}$. Dans le même temps, il prépare, sous le titre «Monographie du Canada», une étude historique portant surtout sur la période coloniale française, étude qui est publiée en dix tranches, entre 1891 et 1894 , dans la revue française, La Science sociale, dirigée par ses maîtres de Paris ${ }^{52}$.

La logique de la démarche intellectuelle nous entrainerait à croire que Gérin se lança en tout premier lieu dans la recherche historique afin de déterminer dans quelles circonstances avaient vu le jour les familles qu'il voulait étudier au point de vue sociologique. "C'est par l'histoire qu'il a abordé ses observations sociales", déclare Falardeau ${ }^{53}$. Rien n'est moins sûr. D'une part, la chronologie des travaux de Gérin ne permet pas de l'affirmer, puisque dès 1886 Gérin a commencé son enquête à Saint-Justin dont on trouve, du reste, des éléments dans les derniers articles de sa "Monographie du Canada ${ }^{54}$. D'autre part, il paraît tout aussi vraisemblable que Gérin ait ajusté l'étude de sa "Nouvelle-France» aux résultats acquis et prévus de son enquête sociologique et surtout à la grille d'interprétation que, pour l'essentiel, il emprunta à Tourville et à Demolins. Dans tout cela, rien que de très naturel et que de très conforme à la démarche toujours plus ou moins à rebours de l'historien.

Que rapporte Gérin de Paris? D'abord l'idée de base de Le Play selon laquelle la famille est non seulement le fondement mais le reflet de la société globale et que, pour une approche de celle-ci, il faut étudier celle-là par la méthode rigoureuse de l'analyse monographique $^{55}$. Il rapporte aussi une typologie des familles et des socié-

51 Ce texte qui connut un certain nombre d'ébauches et de remaniements est reproduit dans Léon Gérin et l'habitant de Saint-Justin, 51-128.

52 Ces études remaniées surtout quant à la division des sujets et de l'expression forment l'ouvrage: Aux sources de notre histoire (Montréal, Fides, 1946).

53 Jean-Charles Falardeau, "Présentation de Léon Gérin et de son ouvre», op. cit., 17. Pour sa part, Hervé Carrier écrit: «Sa première démarche sera de remonter aux sources de la société canadienne et d'analyser les conditions sociologiques de la colonisation française en Amérique. ", Le sociologue canadien Léon Gérin, 1863-195I, 34.

${ }_{54}$ Voir, en particulier, les quatre derniers articles publiés en 1893-1894.

55 «Je ne saurais m'empêcher de penser qu'ici, l'École de Le Play - où Gérin, on le sait, avait trouvé ses maîtres - lui a joué quelque mauvais tour par la primauté (appuyée surtout sur des postulats idéologiques) qu'elle accordait à la 
tés qu'il emprunte à Le Play, revue, corrigée et augmentée par Tourville et Demolins, et qui ne sera pas sans influencer sa conception de la Nouvelle-France.

Il n'est pas facile de résumer, sans la déformer, la thèse qui sous-tend cette typologie de l'École de la science sociale. Au plan des familles, elle oppose, en fin de compte, le type patriarcal ou communautaire au type particulariste. Dans le premier cas, il s'agit d'une famille où règnent la tradition, l'esprit communautaire et patrimonial, l'autoritarisme, le manque d'initiative individuelle. La famille particulariste, au contraire, est marquée par l'esprit d'initiative et d'entreprise, par le caractère individualiste de ses membres, par l'affaiblissement de l'idée patrimoniale et de l'autorité parentale. Contrairement à l'autre, elle favorise la diversification, l'adaptation aux conditions nouvelles, l'essaimage, le progrès ${ }^{56}$.

Selon Tourville, ce sont des conditions géographiques particulières - rendant à la fois difficile par l'exguïté du domaine le soutien de la familile communautaire, mais encourageant la diversification des activités (agriculture, pêcheries, navigation) - qui auraient permis l'éclosion de la famille particulariste. Et le lieu privilégié de cette métamorphose de la tradition en progrès aurait été la côte ouest de la Scandinavie, jadis colonisée par des peuplades germaniques ${ }^{57}$.

Nous passons ainsi du niveau de la cellule familiale à celui de la société particulariste. Déjà caractérisée par les qualités d'initiative et d'ouverture qui marquent les familles qui la composent, cette société (Tourville, Demolins et Gérin emploient le mot race) se distingue par le peu de responsabilités qu'elle confie à la gestion communautaire, au domaine public, à l'Etat. «La vie publique est éliminée, affirme Tourville, c'est le triomphe absolu de la vie privée, se suffisant seule. ${ }^{58}$ »

Bien sûr, la société à formation particulariste ne resta pas confinée à la Scandinavie. À l'époque moderne, c'est en Angleterre, puis aux États-Unis qu'on la retrouve où elle fait éclater son incontestable prédominance. Prenant la relève de Tourville qui a tenté

famille et au groupement.»Fernand Dumont, «L'étude systématique de la société globale canadienne-française", Situation de la recherche sur le Canada français, op. cit.: 286.

s6 Jean-Charles Falardeau, "Présentation de Léon Gérin et de son œuvre», Léon Gérin et l'habitant de Saint-Justin, op. cit., 24-27.

57 Ibid., 28.

$58 \quad$ Ibid., 29. 
d'expliquer la genèse du phénomène particulariste, Demolins publie en 1897 un ouvrage retentissant: À quoi tient la supériorité des AngloSaxons dans lequel il cherche à démontrer, par le système d'éducation, par le comportement dans la vie privée, par la conception de la vie publique, la prépondérance britannique dans le monde. En fait il décrit des attitudes reliées au poids considérable que possède l'initiative privée dans la formation sociale dite particulariste des Britanniques $^{59}$.

Tout cela est assez bien connu, depuis les travaux de Falardeau et de Carrier. Si nous revenons là-dessus avec quelque insistance, c'est pour mieux dégager l'influence profonde que la thèse de la supériorité de la formation particulariste et, surtout, l'exemple qu'en donnait, prétendait-on, le monde anglo-saxon, a pu exercer sur la conception que Gérin s'est faite de la Nouvelle-France et de l'histoire canadienne tout entière, et que l'on retrouve depuis chez un certain nombre de sociologues, en dépit de l'isolement dans lequel a pu s'effectuer le travail intellectuel de Gérin.

Pour Gérin, la sociologie ou selon son expression, le recours «aux procédés les plus rigoureux d'investigation» par «l'observation directe» commence là où l'histoire finit ${ }^{60}$. Or, assez curieusement, l'histoire canadienne se termine à la Conquête. Après avoir consacré six chapitres de sa "Monographie du Canada» à la colonisation de la Nouvelle-France et un septième aux qualités de "l'émigrant percheron» et de «ses similaires», visiblement inféré pour l'essentiel des constatations faites sur le vif à Saint-Justin, il utilise les trois derniers articles pour décrire le comportement de la famille $\mathrm{C}^{* * *}$ qu'il a commencé à étudier sur place à partir de $1886 .{ }^{61}$.

59 Ibid., 30-32. Edmond Demolins, À quoi tient la supériorité des AngloSaxons (Paris, Firmin Didot \& Cie, s.d.), «...le monde anglo-saxon est aujourd'hui à la tête de la civilisation la plus active, la plus progressive, la plus débordante. Il suffit que cette race s'établisse sur un point quelconque du globe pour le transformer en y introduisant, avec une rapidité prodigieuse, les derniers progrès de nos sociétés occidentales - et souvent ces jeunes sociétés arrivent à nous dépasser. Elles nous appellent déjà, avec un certain dédain, le Vieux Monde. Et il faut reconnaître que nous paraissons bien vieux à côté de ces jeunes", préface, ii et iii. Ces quelques lignes contiennent déjà les éléments essentiels d'une interprétation de l'histoire canadienne.

60 «Monographie du Canada, VII - Comment le domaine plein a assuré le maintien de la race», La Science Sociale, 15 (juin 1893): 427.

61 Dans un article intitulé "L'intérêt sociologique de notre histoire au lendemain de la Conquête», Revue trimestrielle canadienne, 1 (mai 1915): 3-14, Gérin tente d'analyser le comportement politique des groupes sociaux de la Conquête à la 
On conçoit que Gérin, comme ses contemporains, ait été pris au dépourvu devant la tâche d'analyser au point de vue social la période historique de l'après-Conquête. Ni l'historiographie déjà orientée du côté des changements constitutionnels et des luttes politiques, ni les documents publiés dont il faisait habituellement ses sources historiques, ne pouvaient lui être d'un grand secours. Mais la suppression d'un siècle d'histoire n'était pas sans conséquences graves. En rattachant directement ses observations sur Saint-Justin à une évolution historique qui s'arrêtait au milieu du dix-huitième siècle, Gérin présumait l'immobilisme des décennies de l'après-Conquête et pavait ainsi - a priori - la voie aux notions de société traditionnelle et de Folk Society dont ses successeurs sociologiques feront un abondant usage. Mais il y a plus. Dans son étude historique de la Nouvelle-France, on ne saurait trouver nulle part l'analyse de l'embryon de société à laquelle se raccorde présumément la famille quasi-patriarcale dont il définit les traits par la méthode sociologique à compter de 1886 . Pour Gérin, la Nouvelle-France, c'est la longue chronique de François $1^{\text {er }}$ à la Conquête, de l'échec répété des agents colonisateurs qui se termine, malgré tout, par le triomphe inespéré, en tout cas inexpliqué, de la paysannerie qui œuvrait dans l'ombre.

Pendant de longues années, l'action sociale de l'habitant, masquée par les hauts faits du seigneur et du coureur de bois, resta inaperçue. Petit à petit, il se [sic] développait pourtant sous l'échafaudage de la bureaucratie coloniale que le gentilhomme français s'était construit à son usage; et quand cet échafaudage se fut écroulé à la suite de l'invasion étrangère, on vit apparaître sous ses débris, les rangs pressés des petits domaines d'habitants, ferme assise de la société canadienne. ${ }^{62}$

On aura compris que pour Gérin, la colonisation est non seulement affaire de peuplement, mais encore de peuplement agricole. Pour lui, le succès de la Nouvelle-France aurait dû être caractérisé par une immigration soutenue de paysans sous la direction de tuteurs - qu'il appelle patrons agricoles - qui auraient veillé à leur installation et à leur progrès sous l'autorité compréhensive d'un État métropolitain favorable à l'initiative individuelle. Or, rien de tel ne se produisit.

Révolution américaine. Il s'agit d'une étude sommaire fondée sur quelques textes des documents constitutionnels et qui ne touche aucunement la réalité même des groupes sociaux.

62 «Monographie du Canada, VII - Comment le domaine plein a assuré le maintien de la race», La Science sociale, 15 (juin 1893): 427. 
François $1^{\text {er }}$ avec qui commencent les efforts de colonisation représente un pouvoir royal «à la fois belliqueux et faible» qui «ne contrôle pas les forces vives de la race». Il n'y a pas à s'étonner «si ce gouvernement besoigneux [sic], sans attaches dans le pays, livré aux dissensions intestines, entraîné par les hasards d'une guerre européenne se montra vacillant dans ses entreprises ${ }^{63}$. Par la suite, pris dans le tourbillon des guerres de religion, "le pouvoir royal sous des chefs médiocres, agités par de puissantes factions, allait être contraint de renoncer à tout projet d'agrandissement, pour ne songer qu'à sa propre conservation. Il sortit de l'arène coloniale: il n'y devait rentrer qu'un siècle plus tard ». ${ }^{64} \mathrm{La}$ tâche de coloniser passe alors aux «gentilshommes français», particulièrement les sieurs de Monts et de Poutrincourt. Mais la gentilhommerie française est pauvre et dépendante. Elle a perdu «sa formation rurale» et aggravé «son caractère guerrier ${ }^{65}$. Elle faillit à son tour.
Ainsi, les gentilshommes, pour avoir déserté la culture, pour s'être engagés dans la voie fausse des carrières militaires ou bureaucratiques, avaient perdu leurs moyens indépendants d'ac- tion. Obligés de compter sur l'appui de l'État, ils n'avaient pu en obtenir qu'un fallacieux privilège commercial ; encore, le pou- voir royal était-il trop instable à cette époque pour leur assurer la jouissance paisible de ce privilège. Ils étaient donc exclus des entreprises de colonisation, exclus parce que leurs associés marchands se lassaient à la longue de leur faire des avances in- fructueuses, exclus, parce que les marchands rivaux, à force de clameurs, les chassaient de leur position privilégiée. ${ }^{66}$

À l'époque de Champlain, ce sont les marchands qui prennent la relève d'un pouvoir royal déficient et d'une noblesse qui a perdu le sens de la vocation agricole. Mais guidés par leur «égoïsme à courte vue», ils ne colonisent pas, ils exploitent ${ }^{67}$. On retrouve ici la notion tenace dans notre historiographie selon laquelle la colonisation n'est pas une exploitation, mais une sorte de sacrifice qu'il faut s'imposer. Pour corriger la situation, Richelieu met sur pied la Com-

63 «Monographie du Canada, I - Les premières tentatives de colonisation", La Science Sociale, 11 (avril 1891): 322-324. - Aux sources de notre histoire, 2224. (Nous citons toujours de préférence le texte de la «Monographie» parce qu'il est, à l'occasion, plus explicite que celui de la refonte de 1946).

64 Ibid.: 331. - Aux sources de notre histoire, 71.

65 Ibid. : 333. - Aux sources de notre histoire, 77-78.

66 Ibid. : 344. - Aux sources de notre histoire, 87-88.

67 "Monographie du Canada, II. - Les premières tentatives de colonisation», La Science Sociale, 11 (juin 1891): 526-550. — Aux sources de notre histoire, 89-112. 
pagnie de la Nouvelle-France qui en dépit de ses bonnes intentions finit par succomber aux intérêts de ses membres; d'une part intérêt «bureaucratique et gentilhomme», d'autre part, intérêt «marchand $" 68$.

La venue au Canada des premiers immigrants à qui on concède des seigneuries et la création de la Communauté des Habitants en 1645 ont pour résultat le transfert dans la colonie des intérêts mercantiles et bureaucratiques et assurent pour un temps la domination du «seigneur fonctionnaire» qui s'y connaît mal en agriculture, qui est beaucoup plus apte «à se faire une carrière dans la magistrature ou dans l'armée» et qui finalement ne dédaignera pas, dans le cadre de la Communauté des Habitants qu'il domine aisément, de tirer le plus possible de la traite des fourrures «aux dépens des vulgaires contribuables ${ }^{69}$. Dans le même temps, d'autres agents de colonisation sont à l'œuvre. Il s'agit des membres des «fondations pieuses", particulièrement de ceux de la Société de Notre-Dame de Montréal. On ne peut les sounçonner d'intérêts mesquins. Mais ils échouent à leur tour par «défaut de sens pratique» et manque de persévérance dans la poursuite de l'œuvre qu'ils ont mise sur pied ${ }^{70}$.

En 1663, l'État métropolitain réapparaît au premier plan sur la scène coloniale. Le titre que donne Gérin à ce chapitre est déjà fort éloquent par lui-même: "Impuissance de Louis XIV à suppléer à l'initiative privée dans la colonisation ${ }^{71}$. Le pouvoir ayant été centralisé et les finances royales rétablies, la monarchie française «entre de plain-pied dans le domaine de la colonisation». Elle s'adjoint "comme rouages principaux destinés à transmettre le mouvement, d'une part une compagnie privilégiée de marchands, de l'autre une hiérarchie de seigneurs terriens ${ }^{72}$. Résultat: double échec, déplore Gérin. La Compagnie des Indes occidentales ne remplit pas la mis-

68 «Monographie du Canada, III. - La première étape de la colonisation administrative», La Science Sociale, 12 (août 1891): 167. — Aux sources de notre histoire, 123.

69 Ibid.: 175 et 182. - Aux sources de notre histoire, 135 et 146.

70 «Monographie du Canada, IV. - La première étape de la colonisation administrative. 2: les fondations pieuses», La Science Sociale, 12 (décembre 1891): 558 et 560. - Aux sources de notre histoire, 177 et 181.

71 "Monographie du Canada, V - Impuissance de Louis XIV à suppléer à l'initiative privée dans la colonisation», La Science sociale, 13 (juin 1892): 519. Dans Aux sources de notre histoire, le titre est devenu «L'effort tenté par Louis XIV».

72 Ibid. : 522-523. - Aux sources de notre histoire, 195-196. 
sion commerciale pour laquelle on l'a créée et «ce que les seigneurs n'avaient pu accomplir par eux-mêmes, ils ne le firent pas davantage avec l'assistance de l'État ${ }^{73}$. La raison de cela: "par suite du défaut d'initiative chez les particuliers, l'ingérence de l'État, tout active qu'elle fût, restait inefficace ${ }^{74}$. Bien plus, elle allait, contre son gré, permettre aux gentilshommes dont elle «n'avait pu faire des agriculteurs... de s'emparer de ce commerce des fourrures et de le constituer sur une large base ${ }^{75}$. Enfin, dernière articulation de l'interprétation que Gérin propose de la colonisation en NouvelleFrance: "La première conséquence de la constitution en grand du commerce des fourrures avait été l'expansion rapide mais superficielle de la colonie; la seconde fut la guerre en permanence ${ }^{76}$ qui, à la fin, se solda par «le triomphe définitif de l'initiative privée dans la colonisation». L'expression est d'autant plus significative qu'elle coiffe comme titre le dernier article de l'exposé historique de Gérin ${ }^{77}$.

C'est finalement, on l'aura compris, la société française tout entière qui est responsable de l'échec répété des essais de colonisation en Nouvelle-France depuis la fin du XVI ${ }^{\mathrm{e}}$ jusqu'au XVIII ${ }^{\mathrm{e}}$ siècle. La philosophie sociale qui sous-tend les thèses de Tourville et de Demolins sert d'explication ultime à Léon Gérin, mué pour un temps en historien. La France que Gérin perçoit à travers Sismondi, c'est d'abord "une vie locale» désorganisée depuis la décadence de la féodalité. L'expression qui revient à plusieurs reprises sous la plume de Gérin implique au départ un défaut d'articulation de la base au sommet de l'édifice social. Le résultat le plus clair est l'impuissance, puis l'atrophie de l'initiative privée. "Ce n'était pas la classe des paysans - excellente, il est vrai, sur certains points, mais partout étroitement limitée et contrecarrée dans son développement - qui pouvait entreprendre d'elle-même le défrichement des forêts vierges du Nouveau Monde», nous dit Gérin ${ }^{78}$. Ce n'était pas non plus «l'ancienne classe dirigeante rurale désorganisée en France par

73 Ibid.: 530. - Aux sources de notre histoire, 206.

74 Ibid.: 527. - Aux sources de notre histoire, 202.

Ibid. : 535. - Aux sources de notre histoire, 215.

76 «Monographie du Canada, VI - Le triomphe définitif de l'intitiative privée dans la colonisation», La Science Sociale, 14 (novembre 1892): 375. - Aux sources de notre histoire, 231.

77 Dans Aux sources de notre histoire, ce titre est devenu «La NouvelleFrance aux mains des Anglais ».

${ }_{78}$ "Monographie du Canada, III. La première étape de la colonisation administrative», La Science sociale, 12 (août 1891): 161-162. 
le militarisme et la bureaucratie ${ }^{79}$. Le gentilhomme français, explique Gérin, «quitte la terre pour vivre à la ville; il se détache du paysan pour suivre la fortune des princes ou se lier au char de l'État. Il devient fonctionnaire et courtisan. Désertion de la culture, charges royales, mœurs urbaines, intrigues de cour, toutes ces choses s'appellent, se provoquent et tendent ensemble à paralyser chez la classe supérieure toute initiative utile, toute action personnelle. ${ }^{80}$ " Reste l'État. À l'époque de François $1^{\text {er }}$, il est à la fois «belliqueux et faible», instable et sans contrôle sur «les forces vives de la race ${ }^{81}$.

Or voyez comme tout s'enchaîne: la monarchie, à ses débuts, au lieu de relever l'individualisme qui déclinait, s'était efforcée, au contraire, d'étouffer toute initiative chez ses sujets français, afin d'augmenter d'autant ses propres attributions. Qu'en était-il résulté ? Lorsque la monarchie, devenue maitresse de tout, voulut à son tour manifester sa force, lorsque, par exemple, elle entreprit de coloniser, l'issue démontra clairement que, sans l'initiative des particuliers, tout succès était éphémère, impossible. Maintenant, cette même monarchie, toujours par suite de l'inaction ou des vices de la classe dirigeante, se trouvait prématurément frappée d'impuissance et voyait sa colonie chanceler au bord de l'abîme. ${ }^{82}$

En parallèle à ce corps social désintégré ou s'étiole l'initiative individuelle, gage de la formation particulariste, Léon Gérin expose avec admiration la situation privilégiée de la société anglaise, là où la colonisation s'organise «en dehors de toute ingérence des pou-

79 «Monographie du Canada, IV - La première étape de la colonisation administrative. 2. Les fondations pieuses», La Science sociale, 12 (décembre 1891): 544-545.

80 «Monographie du Canada, 1 - Les premières tentatives de colonisation», ibid., 11 (avril 1891): 333.

81 Ibid.: 323.

82 "Monographie du Canada, VI - Le triomphe définitif de l'initiative privée dans la colonisation", ibid., 14 (novembre 1892): 390. Ailleurs il écrit: "L'État ne s'est pas constitué en France, comme chez les peuples de race saxonne, notamment aux États-Unis, par l'union libre et pacifique de provinces autonomes en vue de la gestion des intérêts communs. La monarchie française naquit au contraire du dépérissement spontané et, bientôt après, d'une destruction violente des organismes locaux. Deux ordres de faits contribuèrent ainsi à précipiter le dénouement: d'abord la lente décomposition de ces organismes locaux par des causes intimes, puis les agressions répétées du pouvoir royal qui porta les derniers coups aux institutions provinciales et s'établit sur leurs ruines." "Monographie du Canada, III - La première étape de la colonisation administrative», ibid., 12 (août 1891): 163. 
voirs publics, avec le concours et la plupart du temps sous la direction d'un grand seigneur, ou de gentilshommes de la classe moyenne associés dans ce but ${ }^{83}$. Car en Angleterre, contrairement à ce qui s'est passé en France, le seigneur féodal s'est dépouillé petit à petit de «son caractère guerrier pour n'être plus qu'un patron agriculteur ${ }^{84}$. Aussi bien, les entreprises coloniales fondées sur «l'intérêt même des particuliers» et "l'initiative de la classe supérieure des colons», sont-elles assurées d'un succès immédiat qui se traduit au dix-huitième siècle par une supériorité écrasante au point de vue richesse et population ${ }^{85}$. Quand, démesurée, exsangue et sans racines, la Nouvelle-France heurte ces foyers de formation particulariste que sont les colonies anglaises, l'issue du conflit ne fait pas l'ombre d'un doute. En 1763, reprend Gérin avec insistance, «l'initiative privée avait eu raison de la centralisation administrative, sur le terrain même où celle-ci se croyait invincible: sur les champs de bataille ${ }^{86}$.

D'un point de vue strictement historiographique, l'analyse de Léon Gérin ne manque pas d'intérêt. Elle possède des traits qui la placent fort bien dans son contexte de la fin du XIX ${ }^{\mathrm{e}}$ siècle. Outre la conception foncièrement agriculturiste de la colonisation, signalons la suppression claire et nette de tout le $\mathrm{XVIII}^{\mathrm{e}}$ siècle français. Pour Gérin, comme pour beaucoup de ses contemporains, la Nouvelle-France, hormis les guerres, se termine avec un Louis XIV dans la force de l'âge. Par ailleurs, ses préoccupations ne se situent pas au plan national. Ni la langue, ni la foi ne paraissent l'émouvoir outre mesure, et il ne succombe jamais ni au moralisme facile, ni à l'évocation rassurante qui marque l'historiographie canadienne-française de la fin du XIX ${ }^{e}$ et du début du $\mathrm{XX}^{\mathrm{e}}$ siècle. Au contraire, compte tenu de ses prémisses touchant la colonisation agricole, il fait montre d'une rigueur d'analyse et d'une profondeur de réflexion peu communes à l'époque ${ }^{87}$.

\footnotetext{
83 «Monographie du Canada, I - Les premières tentatives de colonisation», ibid., 11 (avril 1891): 332.

84 Ibid. : 332-333.

85 «Monographie du Canada, VI - Le triomphe définitif de l'initiative privée dans la colonisation», ibid., 14 (novembre 1892): 387.

86 Ibid.: 392.

87 Il excelle en particulier à démêler les intérêts des divers groupes sociaux en présence, sans se laisser distraire par des considérations pieuses ou patriotardes. En ce sens, il tranche nettement sur ses contemporains.
} 
Mais si les caractères nationaux, tels qu'on les évaluait à la fin du XIX ${ }^{\mathrm{e}}$ siècle l'intéressent peu, c'est peut-être qu'ils ne sont pour lui que les affleurements culturels d'une réalité beaucoup plus profonde que l'on perçoit au niveau de l'organisation sociale et que lui et ses collègues de Paris désignent sous le terme assez ambigü de race. Dans cette perspective axée sur la formation communautaire ou particulariste et dont l'explication est d'ores et déjà toute contenue dans les allégations pseudo-historiques de Tourville et de Demolins, la démarche de Gérin se situerait non pas en aval, mais en amont du courant historiographique nationaliste de la fin du XIX ${ }^{\mathrm{e}}$ siècle. En d'autres termes, sous le couvert d'études socio-historiques qui paraissent avancées pour l'époque, on assisterait à la simple application au cas canadien d'une classification simpliste de ce qu'on appelait à l'époque les races, classification fondée non sur des critères psychologiques ou physiologiques, mais sur des critères sociaux $^{88}$.

Assez curieusement, malgré l'originalité de la démarche, Gérin finit par rejoindre un courant fort répandu à l'époque, qui n'a touché que superficiellement l'historiographie canadienne-française, mais qui affecte en profondeur l'historiographie anglo-saxonne du Canada et des États-Unis. Il s'agit du courant parkmanien fondé lui aussi sur la prédominance des sociétés, des institutions et de la mentalité anglo-saxonnes et qui, aujourd'hui encore, n'en finit pas, malgré les nuances dont on le pare, de nous montrer la Nouvelle-France comme un étrange objet de musée. Dans quelle mesure cette pensée qui, à travers Gérin, unit Demolins à Parkman, a-t-elle affecté la vision historique de nos sociologues des années 1960 et en particulier leurs conceptions pancanadianistes? Il n'est pas facile de le préciser. En revanche il semble assez clair que c'est la convergence jusqu'à un certain point fortuite entre la pensée de l'École de la science sociale de Tourville et de Demolins et celle de l'historiographie anglo-saxonne touchant le Canada qui a permis aux sociologues de Chicago de prendre la relève de Gérin sans que le support historique des analyses sociologiques ne paraisse avoir été modifié.

Or, chez Gérin, ce support historique se réduit finalement à fort peu de choses. Après avoir décrit longuement l'échec des agents

88 Dans un article où il se porte à la défense du livre de Demolins sur la supériorité anglo-saxonne, Gérin est amené à exposer clairement la différence à faire entre les critères psycho-physiologiques (qu'il rejette) et les critères sociaux de distinction des races. Voir «M. Demolins et la science sociale. Réponse aux critiques", La Revue canadienne, 40, no 4 (1er avril 1904): 338-358. 
colonisateurs jusqu'à Louis XIV, après avoir escamoté le XVIII ${ }^{\mathrm{e}}$ siècle et narré le triomphe de l'initiative privée par la Conquête anglaise, il nous brosse un tableau de l'immigrant percheron, prototype du paysan français qui, contre vents et marées, réussit là où le seigneur, le gentilhomme, le marchand, la compagnie, la communauté religieuse et l'État ont failli ${ }^{89}$. La faiblesse du procédé et de la méthodologie sautent aux yeux: surestimation évidente de l'immigration percheronne, connaissance superficielle de la société rurale du Perche, fondée sur l'apport de quelques monographies obscures; utilisation abusive de l'analogie touchant les conditions du milieu naturel de vie de l'immigrant de part et d'autre de l'Atlantique; extension commode des qualités du percheron aux immigrants "similaires»; surtout, projection rétrospective au $\mathrm{XVII}^{\mathrm{e}}$ siècle des caractéristiques (famille-souche, domaine plein, frontières sociales du rang et de la paroisse) du milieu paysan, évaluées sur place à la fin du $\mathrm{XIX}^{\mathrm{e}}$ siècle.

Nous n'affirmons pas, bien sûr, que les concordances que Gérin établit entre le $\mathrm{XIX}^{\mathrm{e}}$ et le $\mathrm{XVII}^{\mathrm{e}}$ siècle, entre les résultats de l'analyse sociologique et de la démarche historique, sont nécessairement fausses, mais plutôt qu'elles ne sont prouvées, ni aujourd'hui, ni, cela va de soi, à son époque. Il faudra, pour y voir clair, étendre à toute la colonie et sur toute la durée de la Nouvelle-France (sans parler des décennies qui suivent et qui se situent, dit-on, dans «un Québec qui bougeait») une analyse de la famille paysanne qui est à peine ébauchée.

Aussi bien, tous les raccordements par voie historique que Gérin et ses successeurs hantés par les conditions d'accès du Canadien français à l'ère industrielle ont pu tisser vers le passé de la Nouvelle-France restent-ils suspects. Ces raccordements utilisent comme point d'ancrage une société rurale de jadis dont l'historiographie canadienne n'a pu fournir jusqu'à maintenant que des imageries. Par-dessus tout, ils présupposent a priori et implicitement que cette société rurale constituait la part essentielle, vitale, durable de la Nouvelle-France: ce qui implique un concept diminué, étroitement univoque de la colonisation et dont l'historiographie s'est éloignée depuis déjà longtemps.

89 «Monographie du Canada, VII - Comment le domaine plein a assuré le maintien de la race», La Science sociale, 15 (juin 1893): 426-446. - Aux sources de notre histoire, 255-261. 


\section{La «Folk Society» et son support historique}

Dans un texte polémique placé en avant-propos de ses Études sur le Canada français, le sociologue Philippe Garigue s'en prend à l'utilisation abusive, dans l'étude de la société québécoise, du concept de Folk Society popularisé par Redfield, répandu par des sociologues de Chicago et qui «semble avoir imprégné pratiquement tous les travaux sociologiques consacrés au Canada français ${ }^{90}$. Selon $\mathrm{Ga}-$ rigue, ce concept qui désigne une communauté traditionnelle, repliée sur elle-même, fortement unie et dont l'horizon ne dépasse guère les limites du milieu familial, ne résiste pas à l'examen. «En tant qu'il est une hypothèse, dit-il, un tel concept n'apparaît étayé sur aucune donnée empirique; en tant qu'il est une catégorie mentale, de "type idéal», il revêt une signification méta-sociologique qui ne saurait manquer de défigurer l'analyse de la réalité sociale qu'il est censé clarifier. ${ }^{91}{ }^{\text {}}$ Plus loin, il ajoute: «soi-disant proposé comme l'expression d'un rapport fonctionnel [ce concept] n'a jamais fait l'objet d'une confrontation sérieuise àvec les réalités historiques du Québec». Enfin, déclare-t-il, les sociologues de Chicago "construisent une histoire des changements sociaux au Canada français sans du tout tenir compte de l'apport des historiens ${ }^{92}$.

Les sociologues que vise Garigue sont d'abord Horace Miner, élève de Redfield qui, en 1939, fait paraître St. Denis, a FrenchCanadian Parish ${ }^{93}$ et Everett C. Hughes qui publie, en 1942, French Canada in transition ${ }^{94}$, traduit par Jean-Charles Falardeau, trois ans plus tard, sous le titre: Rencontre de deux mondes. La crise d'industrialisation du Canada français ${ }^{95}$. Mais d'autres sociologues

90 Philippe Garigue, Études sur le Canada français (Montréal, Faculté des sciences sociales, économiques et politiques, Université de Montréal, 1958), 7.

91 Ibid., 7.

92 Ibid., 8.

93 Horace Miner, St. Denis, a French-Canadian Parish (The University of Chicago Press, 1939). L'ouvrage fut réédité en 1963 avec un nouvel avant-propos où Miner répond aux attaques de Garigue.

94 Everett C. Hughes, French Canada in transition (The University of Chicago Press, 1942). Hughes séjourne à l'Université Laval en 1942-1943. Il rédige un «Programme de recherches sociales pour le Québec» qui constitue, selon Falardeau, une invitation à reprendre «la pratique monographique de Léon Gérin». Voir JeanCharles Falardeau, "Antécédents, débuts et croissance de la sociologie au Québec», Recherches sociographiques (mai-décembre 1974): 145.

95 Everett C. Hughes, Rencontre de deux mondes. La crise d'industrialisation du Canada français (Montréal, Éditions Lucien Parizeau, 1945). Une réédition est parue en 1972 (Éditions du Boréal Express) avec une "présentation» de JeanCharles Falardeau. 
comme Falardeau, Rioux, Guindon, sont ou se sentiront impliqués par les formules, parfois imprécises mais volontiers généralisatrices, de Garigue.

Ce qui est particulièrement intéressant pour nous c'est que, parti du concept de Folk Society (ou de Folk-Urban Continuum qui en est la transposition dans le devenir) Garigue cherche à y rattacher l'œuvre de Léon Gérin sur laquelle «la notion de Folk Society n'a manifestement exercé aucune influence, mais dont les généralisations ne laissent pourtant de concorder sur plusieurs points avec celles de Miner ${ }^{96}$.

Parmi les généralisations formulées par Gérin, la plus fondamentale sur laquelle repose toute sa théorie, est celle qui veut que le Québec rural se compose d'un certain nombre de groupes familiaux, jouissant de statuts à peu près égaux et possédant chacun la même étendue de terre. L'entretien de la propriété foncière et sa transmission intégrale par un héritier unique constitueraient l'idéal prédominant des membres de la famille. Voilà à qui serait, selon Gérin, le pivot de la société rurale, son centre de gravité, auquel tout le reste viendrait s'articuler. Non seulement pareille généralisation a-t-elle été acceptée par les sociologues de Chicago, mais elle leur a servi de point de départ pour démontrer que le Canada français «traditionnel» ne pouvait changer que sous le coup d'innovations introduites par l'élément culturel anglais. D'autres sociologues adhérèrent eux aussi à cette façon de voir, contribuant de la sorte à entretenir l'impression que les changements sociaux survenus dans la société canadienne-française ne pouvaient être autre chose que des contrecoups d'un dynamisme d'origine anglo-saxonne. Ici encore il apparaît pour le moins probable qu'un subtil parti pris soit intervenu dans le choix des théories pour l'infléchir dans un sens assez peu réaliste. ${ }^{97}$

Quoi qu'il en soit du «parti pris», ce qui ressort de l'analyse de Garigue, c'est que depuis Léon Gérin à la fin du XIX ${ }^{\mathrm{e}}$ siècle jusqu'aux premiers travaux de Marcel Rioux dans les années 1950, en passant par les études des sociologues de Chicago, toutes les recherches portant sur la société québécoise avec finalement, comme borne repère, le passage à l'ère industrielle, sont parties de préalables historiques semblables où l'on retrouve comme points forts: une société rurale à l'origine, essentiellement axée sur une famille qui tend à

96 Philippe Garigue, Études sur le Canada français, op. cit., 10.
97 Ibid., 11. 
l'équilibre entre ses composants et son patrimoine dont l'intégrité doit être maintenue à travers les générations; une famille dont l'ambition, l'activité, l'éducation se limitent à ce que peuvent offrir les premières patries du rang et de la paroisse; une famille enfin que met en danger l'initiative du particularisme anglo-saxon.

Nous avons vu les fondements historiques des positions sociologiques de Léon Gérin. Si nous examinons, dans un même tout, l'ouvrage de Miner, nous constatons que l'auteur a bien lu L'habitant de Saint-Justin, mais qu'il ignore la Monographie du Canada. En revanche, il s'est largement inspiré, dans son analyse du passé, de Parkman et de Bracq ${ }^{98}$. C'est déjà énumérer ce que nous pouvons trouver dans le raccourci historique qui couvre une vingtaine de pages: une étrange société - dont on sent bien à travers l'ouvrage qu'elle est l'objet d'étude rêvée pour le chercheur à la fois étranger, scientifique et complaisant - façonnée de traditionalisme ${ }^{99}$, d'immobilisme ${ }^{100}$ et de religiosité ${ }^{101}$. Très tôt, du reste, dans ses quelques pages où les faits de colonisation se résument à des données succinctes sur l'immigration et le système seigneurial, l'image globale de la Nouvelle-France se rétrécit aux limites de la paroisse de SaintDenis dont on ne quitte à peu près plus les horizons, depuis la fin du XVII ${ }^{\mathrm{e}}$ siècle jusqu'en 1936.

Il n'y aurait rien à redire à un rappel historique aussi sommaire, si l'analyse sociologique de l'auteur n'aboutissait pas à des conclu-

98 Jean Charlemagne Bracq, The Ev'olution of French Canada (New York, The Macmillan Company, 1924). Cet ouvrage, traduit en français en 1927, est une ouvre de vulgarisation pour lecteurs américains. On y lit que pour la Nouvelle-France, "the writer usually refers to documents not accessible to him, but used by trustworthy writers» (Introduction, p. vii). Qu'il suffise d'ajouter que l'un des deux chapitres consacrés à la période française s'intitule: "Theocratic formation of French Canada».

99 «As a reminder to every French child that priority rights in Canada are his, rustic crosses bearing the fleurs-de-lis escutcheon of France stand before schools in all the parishes of the province. They represent the cross planted by Jacques Cartier in Gaspé four hundred years ago, when he first laid eyes on the St. Lawrence.» Horace Miner, op. cit, 1.

100 «The fundamental structure of the rural parish goes back to the earliest days...»Ibid., 1.

101 "The religious aspect of the settlement cannot be too greatly emphasized... The whole colonial enterprise was charged with the crusading spirit... Miraculous intervention from heaven was not unusual... This general acceptance of the miraculous as a natural result of prayer and religious activity continues only slighty diminished to the present day. »Ibid., 2. 
sions qui, la plupart évoquent plus qu'elles ne décrivent directement une société tournée vers un passé lointain et figée dans le temps. Dans ce cas - comme dans celui de Gérin - le passé auquel explicitement ou implicitement on fait sans arrêt référence, acquiert une valeur démonstrative considérable dans l'exposé sociologique, et il paraîtrait élémentaire que l'auteur s'en assure dès le départ la connaissance la plus approfondie possible. Or, le moins qu'on puisse dire, c'est que Miner n'y a vraiment pas épuisé ses énergies. The Old Regime in Canada et quelques ouvrages satellites ont paru lui suffire. Dès lors, la tentation devenait forte dans ce vide de coordonnées historiques d'improviser largement sur le thème de la tradition paysanne que suggère l'ouvrage à chaque détour. Redfield qui en signe l'introduction y succomba avec virtuosité. Plus tard, en 1963, Miner répondant aux critiques de Garigue écrira: ${ }^{102}$

Although the author was a student of Redfield, the analysis of St. Denis was made along ethnological and structural-fonctional lines, strongly influenced by the teaching. of A. R. RockliffeBrown. Culture change was conceptualized as resulting from social structure forces and from diffusion, not as a shift away from a folk type of culture. It is in the widely quoted Introduction to the study, written by Robert Redfield, that the data from St. Denis were put into the context of peasant culture.

Tout en admettant que Miner n'a pas tort, il faut convenir que son livre, par l'absence d'arrière-fond historique, invite à l'interprétation qu'en donne Redfield qui, au total, ne fait que projeter dans un passé aux limites imprécises - antérieures en tout cas à la prise de possession du territoire par les Français - les caractéristiques observées dans une paroisse du $\mathrm{XX}^{\mathrm{e}}$ siècle: société paysanne «qui ressemble aux peuples primitifs» et qui vit «selon des règles et des valeurs collectives qui sont enracinées dans la tradition», société où le prêtre «détermine ce qui est bien et ce qui est mal... où domine la famille, comme c'est le cas dans bien d'autres sociétés plus primitives, extérieures au monde européen $»{ }^{103}$ Derrière ces phrases simplistes, c'est tout un passé sans nuances qui se profile.

En 1945, Everett C. Hughes publie French Canada in transition. Il s'agit de l'analyse sociologique d'une petite ville du Qué-

102 Avant propos à l'édition de 1963, VI et VII.

103 Nous citons la version française de l'introduction de Robert Redfield à l'ouvrage de Miner parue dans Marcel Rioux et Yves Martin, éd., La société canadiennefrançaise (Montréal, Hurtubise HMH, 1971), 69-73. 
bec, Cantonville, qui constitue dans l'esprit de Hughes, «le point médian du continuum rural-urbain ${ }^{104}$. Il serait facile de démontrer que, pour Hughes, le terme $a$ quo de ce continuum, c'est-à-dire la Folk Society, n'est pas remis en question et qu'il a été au moins partiellement décrit par Gérin et Miner dont l'avant-propos de l'ouvrage fait l'éloge. Le deuxième chapitre qui situe la société rurale dans «un fond de scène» servant de point de départ répète sur la famille et la paroisse les données que l'on trouve dans Le type économique et social des Canadiens et St. Denis, a French-Canadian Parish, y compris la phrase devenue célèbre où Gérin supprimant les nuances qu'il avait lui-même apportées, finit par parler d'un type unique de famille rurale quasi auto-suffisante et dont la plus haute ambition est de transmettre intact le patrimoine familial à l'un de ses enfants. ${ }^{105}$

Bref, il paraît évident que de Gérin à Hughes, en passant par Miner et la touche simplificatrice de Redfield, on réduisait de plus en plus la complexité de la société d'origine pour faire entrer celleci dans le cadre étroit de la Folk Society, ce qui permettait d'y aller avec plus d'aise dans l'analyse du continuum rural-urbain. Dans cette aventure d'un demi-siècle, on ne semblait guère s'émouvoir de l'image inventée et sans doute caricaturale qu'on donnait du Canada français des XVIII ${ }^{\mathrm{e}}$ et $\mathrm{XIX}^{\mathrm{e}}$ siècles. À la suite de l'initiative de Gérin, renforcée par la vision parkmanienne des savants de Chicago, la Nouvelle-France des sociologues n'était plus que familles paysannes traditionnelles, rangs et paroisses.

C'est dans la foulée de Gérin et des sociologues de Chicago que Jean-Charles Falardeau s'intéressa particulièrement à la paroisse. L'étude qu'il publia sur la paroisse canadienne-française au XVII ${ }^{\mathrm{e}}$ siècle, mises à part les données à caractère juridique, est un bel exemple d'une rétroprojection au temps de Louis XIV de la valeur d'unité sociale que pouvait constituer la paroisse à l'époque des analyses de Gérin et de Miner. ${ }^{106}$ Jusqu'à maintenant aucun travail historique n'a encore démontré qu'il en était ainsi au XVII ${ }^{\mathrm{e}}$ siècle.

104 Everett C. Hughes, Rencontre de deux mondes (réedition de 1972), "Présentation" de Jean-Charles Falardeau, X.

105 Ibid., 25.

106 Jean-Charles Falardeau, «La paroisse canadienne-française au XVII ${ }^{\mathrm{e}}$ siècle», article reproduit dans Marcel Rioux et Yves Martin, éd., La société canadiennefrançaise: 33 à 43. Voir aussi du même auteur: «Rôle et importance de l'Église au Canada français», dans ibid. : 349-361. 
En 1962, faisant le bilan des recherches sur le milieu rural, Gérald Fortin écrit: "L'image de Saint-Justin, Rioux la retrouvait lui aussi à l'île Verte durant les années 40 . Nous-mêmes, nous l'avons retrouvée en essayant de reconstituer la vie sociale de Sainte-Julienne (Dorchester) durant les années 30.»Dans le même texte il avouait qu'il lui semblait tout à fait naturel que ce soit le milieu rural «numériquement majoritaire et idéologiquement primordial qui ait attiré l'attention de la sociologie naissante». Puis il ajoutait: «On peut même comprendre qu'il y ait eu tendance chez les pionniers à généraliser les résultats de leur recherche à l'ensemble de la société canadienne-française, à identifier notre culture traditionnelle à la culture observée dans un petit nombre de paroisses rurales. " ${ }^{107}$ C'était là admettre au moins partiellement le bien-fondé des remarques de Garigue et convenir que le postulat de la Folk Society avait eu l'effet néfaste qu'on retrouve souvent dans la démarche scientifique d'enrégimenter les chercheurs et d'étendre induement son emprise sur une réalité sociale ${ }^{108}$ qu'on commençait à croire beaucoup plus complexe.

Mais d'autres sociologues visés par Garigue réagiront à ces propos parfois avec âpreté. Marcel Rioux est ainsi amené à défendre l'interprétation historique sur laquelle repose l'utilisation du concept de Folk Society au Canada, en décrivant une NouvelleFrance où l'ajustement de l'immigrant au milieu est minimisé en sorte que les paysans sont «des paysans français qui vivent en Canada» (thèse à la fois de Gérin et de Parkman); où les établissements urbains «ne devaient pas être bien différents» des établissements ruraux; où le rang et la paroisse constituent les premières unités sociales. ${ }^{109}$ Et Rioux d'appuyer ses affirmations sur Gérin, Groulx, Henripin, Deffontaines et Falardeau. Sauf pour Henripin

107 Gérald Fortin, «L'étude du milieu rural», dans Situation de la recherche sur le Canada français, ouvrage réalisé par Fernand Dumont et Yves Martin (Québec, les Presses de l'Université Laval, 1962): 105 à 116.

108 Comme il arrive fréquemment en pareil cas, l'expression se meut en passepartout commode: "À la fin du régime français, la colonie est déjà ainsi orientée vers la "Folk Society», déclare Fernand Dumont, dans «Idéologie et conscience historique dans la société canadienne-française du XIX ${ }^{\mathrm{e}}$ siècle», rapport publié dans France et Canada français du XVI $I^{\circ}$ au $X X^{\prime}$ siècles, édité par Claude Galarneau et Elzéar Lavoie (Québec les Presses de l'Université Laval, 1966); 273.

109 Marcel Rioux, "Note sur le développement socio-culturel du Canada français», dans Marcel Rioux et Yves Martin, éd., La société canadienne-française: 173187. 
que d'ailleurs on interprète mal, on nage en pleine pétition de principe. ${ }^{110}$ Nous retrouvons une logique à peu près semblable dans un texte de Hubert Guindon publié en 1960 et intitulé «Réexamen de l'évolution sociale du Québec». ${ }^{111}$ Guindon écrit:

On peut prouver que les accusations d'ignorance de l'histoire sont sans aucun fondement. Miner et Hughes seraient tombés sans le vouloir dans le traquenard préparé par Gérin en raison de leur ignorance de l'histoire du Canada français. A fortiori, ce serait également à l'ignorance de cette histoire que Gérin dut de formuler son hypothèse de base. Mais Gérin connaissait l'histoire de l'ancienne société canadienne-française puisqu'il y consacra un livre.

La dernière phrase se passe de commentaires! Guindon essaie ensuite d'établir que Gérin, contrairement aux dires de Garigue, percevait la société de la Nouvelle-France comme société marchande. Or, une lecture attentive de l'œuvre historique de Gérin montre pourtant qu'il s'agit plutôt de l'étude d'agents colonisateurs qui tous, dans l'esprit de l'auteur, sauf les paysans, ont prévariqué. Nulle part on y trouve l'analyse d'une société qu'elle soit marchande ou paysanne.

Ignorance de l'histoire, clamait Garigue à l'endroit de ses collègues. La formule est trop brutale. Il est presque aussi impossible d'ignorer l'histoire que de la connaitre. Parlons plutôt d'interprétations archaïques issues du XIX $\mathrm{XI}^{\mathrm{e}}$ siècle et que les sociologues étaient peut-être les derniers au milieu du $\mathrm{XX}^{\mathrm{e}}$ à vouloir maintenir vivantes.

110 Rioux cite Henripin: «En 1721, 82 paroisses groupaient cette population riveraine, 48 sur la rive gauche, et 34 sur la rive droite. Trois agglomérations présentaient alors un caractère urbain: Québec, Montréal, Trois-Rivières.» Et Rioux conclut: "Donc, dans la Nouvelle-France de 1721, on compte 82 établissements dont trois à caractère urbain. On peut dire, à coup sûr, que ces trois établissements ne devaient pas être bien différents des autres" (175-176). Drôle de conclusion! Ailleurs, Rioux, comme beaucoup d'autres sociologues, s'appuie sur l'article de Pierre Deffontaines. intitulé «Le rang, type de peuplement rural du Canada français», paru en 1953 et reproduit partiellement dans La société canadienne-française : 19 à 32. Or, ce qui importe aux tenants de la "Folk Society", c'est le caractère social du rang. Quand Deffontaines aborde cet aspect sous le sous-titre "Autonomie sociale du rang", il fait référence à ... Falardeau.

111 Hubert Guindon, "Réexamen de l'évolution sociale du Québec», dans Marcel Rioux et Yves Martin, éd., La société canadienne-française: 149-171. À noter chez Guindon une sorte de rajustement de tir qui montre une certaine influence de l'historiographie de l'école de Montréal. Pour lui, la Nouvelle-France est une entreprise commerciale à base de féodalisme (ce qu'elle n'était pas chez Gérin, ni chez Miner). C'est la Conquête qui fit apparaître la «Folk Society». 
Pour sa part, Garigue va s'efforcer de démontrer que contrairement à ce qu'avaient soutenu, depuis Gérin, les sociologues qui parlaient volontiers de crise de l'industrialisation et de culture canadienne-française sociologiquement inadéquate, «l'urbanisation des régions rurales du Québec s'est poursuivie sans susciter de graves frictions et tensions parce que les façons de vivre étaient dans l'ensemble les mêmes ». ${ }^{112}$ Entendons par là que «l'évolution des structures sociales a empêché que se crée une culture proprement rurale» 113 dans un milieu où à l'origine - en Nouvelle-France - la société avait un «caractère urbain et commercial»"114. On le voit, l'arrière-fond historique s'est passablement rajeuni par rapport à ce qu'il était chez les disciples de Gérin et les tenants de la Folk Society. Garigue s'appuie sur Frégault et en particulier sur La société canadienne sous le régime français. Il nous propose une NouvelleFrance qui, à ses débuts, "se composait d'une série d'établissements commerciaux et missionnaires qui devinrent éventuellement des villes» ${ }^{115}$; où les immigrants «n'apportèrent peu ou rien des institutions de la France paysanne ${ }^{116}$; où ni la seigneurie ni le rang, ni même la paroisse (avant le XIX ${ }^{\mathrm{e}}$ siècle) ne constituaient des unités d'intégration sociale ${ }^{117}$; où finalement «en 1754 un Canadien français sur quatre vivait dans une des trois villes de la colonie et 4,000 hommes, sur une population totale de 55,000 âmes (hommes, femmes, enfants) travaillaient en permanence à la traite des fourrures. C'était l'époque où les frontières commerciales s'allongeaient sans cesse et où prévalait la mentalité de la «frontière», et où, dans les centres urbains de plus en plus importants, s'imposait une culture religieuse franchement urbaine ${ }^{118}$ ».

112 Philippe Garigue, «Évolution et continuité dans la société rurale canadiennefrançaise », dans Marcel Rioux et Yves Martin, éd., La société canadienne-française : 147. Il s'agit de la version française d'un texte paru en 1957 sous le titre: "Change and continuity in Rural French Canada» et reproduit dans Études sur le Canada français : 17-28.

113 Ibid.: 147

114 Philippe Garigue, «Avant-propos», Études sur le Canada français : 8.

115 Philippe Garigue, «Évolution et continuité dans la société rurale canadiennefrançaise », ibid.: 138.

116 Ibid.

117 Ibid.: 139-141.

118 Philippe Garigue, «Avant-propos», Études sur le Canada français: 8-9. 
Garigue a lu Frégault, c'est évident, mais avec une imagination furibonde. ${ }^{119} \mathrm{Il}$ propose comme antithèse à la société rurale traditionnelle perçue par Parkman et Gérin, une société frontiériste que les raccourcis de la preuve ont complètement déformée et dont, certes, l'image n'existe nulle part dans notre historiographie. Du reste, eût-il suivi fidèlement Frégault qu'il eût compris que $L a$ société canadienne sous le régime français est une hypothèse d'explication globale fort enrichissante mais qui reste encore indémontrée. Tout compte fait, dans ses prémisses, Garigue commettait lui-même la faute qu'il reprochait à ses adversaires sociologues: celle d'établir imprudemment les assises historiques de leurs explications sociologiques.

\section{CONCLUSION}

Au tournant des années 1960, les sociologues furent parmi les premiers à s'inquiéter "de l'interprétation monolithique qu'ont donnée les historiens de l'histoire du Canada français».120 Mais à ce moment on avait l'inquiétude nerveuse, trépignante et peu clairvoyante des veilles de «révolution». Il avait suffi que l'historiographie même récente fût contruite sur le concept de nation pour qu'on la classât parmi les «valeurs» à rejeter. Pour beaucoup de sociologues, le nationalisme, c'était alors la tradition «le refus de l'insdustrialisation», ${ }^{121}$ la négation du présent et de l'avenir. Il fallait, dès lors, dénoncer une historiographie dont le caractère systématique paraissait «l'éloigner fatalement de nos situations et de nos questions d'aujourd'hui». ${ }^{122}$ Ils s'y employèrent sans toujours y mettre ni la profondeur ni les nuances qu'il aurait fallu. Peu importe: ils al-

119 Dans une note de son article: «Évolution et continuité dans la société rurale canadienne-française» dit expressément qu'il s'appuie pour l'analyse de la société en Nouvelle-France sur les deux ouvrages de Frégault: La civilisation de la NouvelleFrance et La société canadienne sous le régime français. Il ne semble pas avoir perçu le caractère antithétique de ces deux œuvres.

120 Marcel Rioux, «Notes sur le développement socio-culturel du Canada français», dans Marcel Rioux et Yves Martin, éd., La société canadienne-française: 173. rain: 248

121 Jean-Charles Falardeau, «Perspectives», Essais sur le Québec contempo-

122 Fernand Dumont, «L'étude systématique de la société globale canadiennefrançaise», dans France et Canada français du XVI au XX $X^{e}$ siècles, édité par Claude Galarneau et Elzéar Lavoie, op. cit.: 283. 
laient malgré tout contribuer à ce qui, difficilement, deviendra un renouveau historiographique.

Mais, paradoxalement, plusieurs de ces sociologues qui s'émouvaient tant de voir le national étouffer le social utilisaient dans leur tâche professionnelle un cadre de références historiques marqué au coin du racisme sociologique (Gérin) ou du racisme psychologique (Parkman) et que l'historiographie canadienne-française avait abandonné depuis un demi-siècle.

Il est intéressant de noter qu'à côté d'eux d'autres spécialistes des sciences humaines, moins affectés par la lignée Gérin-MinerHughes, se faisaient du passé une image tout à fait différente. Qu'on relise, par exemple, certains articles parus au cours de la décennie 1950-1960 de Maurice Tremblay et d'Albert Faucher. ${ }^{123}$ On y découvre une Nouvelle-France qui n'est plus celle de Gérin ou le Parkman. C'est la Nouvelle-France d'Innis et de Creighton qui ne paraît pas avoir distrait outre mesure les sociologues de la Folk Society.

D'un point de vue strictement historiographique, ce que nous avons appelé «la voie des sociologues» aboutit finalement à la remise en question de l'histoire nationaliste non par la force du raisonnement, ni par la force de l'exemple, mais plutôt par le cri bien orchestré de distinguer le social du national, et par un certain nombre de questions pertinentes ${ }^{124}$ qui continuaient de rester sans réponse. De son côté, l'école de Montréal avait rajeuni l'histoire nationaliste, y avait insufflé une logique impeccable, l'avait rendue «existentielle», mais tendues à l'extrême les coordonnées de la problématique devenaient inquiétantes. Elles ne permettaient qu'une solution globale du genre panacée qui guérit tous les maux. Elles n'offraient ni ré-

123 Maurice Tremblay, «Orientations de la pensée sociale», Essais sur le Québec contemporain: 193-208. - Albert Faucher, «La dualité canadienne et l'économique: tendances divergentes et tendances convergentes ", Mason Wade, éd., La dualité canadienne. Essais sur les relations entre Canadiens français et Canadiens anglais, 222-238. On retrouvera d'Albert Faucher plusieurs textes de cette époque ou même antérieurs à cette époque, dans Albert Faucher, Histoire économique et unité canadienne (Montréal, Fides, 1970).

124 On trouve ces questions dans plusieurs textes de type bilan que produisent les sociologues à cette époque. Voir, par exemple, de Jean-Charles Falardeau, «Perspectives», Essais sur le Québec contemporain: 239-257 ou encore plusieurs écrits de Fernand Dumont dont «L'Étude systématique de la société globale canadienne-française», Situation de la recherche sur le Canada français: 277-292. 
ponses ni même perspectives de réponses aux questions valables des sociologues. sourds.

D'autres historiens tenteront de mettre fin à ce dialogue de 